NASA/TM-2018-219806

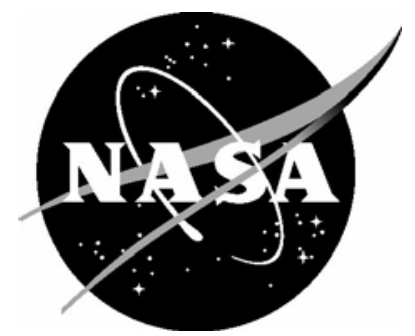

\title{
Acoustic Radiation Pressure
}

\section{John H. Cantrell}

Langley Research Center, Hampton, Virginia 


\section{NASA STI Program . . . in Profile}

Since its founding, NASA has been dedicated to the advancement of aeronautics and space science. The NASA scientific and technical information (STI) program plays a key part in helping NASA maintain this important role.

The NASA STI program operates under the auspices of the Agency Chief Information Officer. It collects, organizes, provides for archiving, and disseminates NASA's STI. The NASA STI program provides access to the NTRS Registered and its public interface, the NASA Technical Reports Server, thus providing one of the largest collections of aeronautical and space science STI in the world. Results are published in both non-NASA channels and by NASA in the NASA STI Report Series, which includes the following report types:

- TECHNICAL PUBLICATION. Reports of completed research or a major significant phase of research that present the results of NASA Programs and include extensive data or theoretical analysis. Includes compilations of significant scientific and technical data and information deemed to be of continuing reference value. NASA counter-part of peer-reviewed formal professional papers but has less stringent limitations on manuscript length and extent of graphic presentations.

- TECHNICAL MEMORANDUM. Scientific and technical findings that are preliminary or of specialized interest, e.g., quick release reports, working papers, and bibliographies that contain minimal annotation. Does not contain extensive analysis.

- CONTRACTOR REPORT. Scientific and technical findings by NASA-sponsored contractors and grantees.
- CONFERENCE PUBLICATION. Collected papers from scientific and technical conferences, symposia, seminars, or other meetings sponsored or co-sponsored by NASA.

- SPECIAL PUBLICATION. Scientific, technical, or historical information from NASA programs, projects, and missions, often concerned with subjects having substantial public interest.

- TECHNICAL TRANSLATION.

English-language translations of foreign scientific and technical material pertinent to NASA's mission.

Specialized services also include organizing and publishing research results, distributing specialized research announcements and feeds, providing information desk and personal search support, and enabling data exchange services.

For more information about the NASA STI program, see the following:

- Access the NASA STI program home page at http://www.sti.nasa.gov

- E-mail your question to help@sti.nasa.gov

- Phone the NASA STI Information Desk at 757-864-9658

- Write to:

NASA STI Information Desk

Mail Stop 148

NASA Langley Research Center

Hampton, VA 23681-2199 
NASA/TM-2018-219806

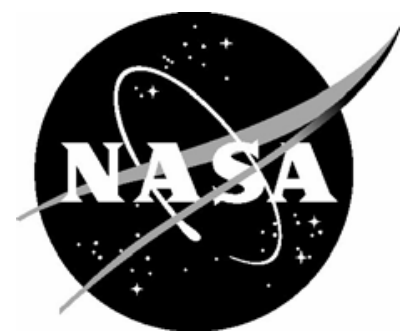

\section{Acoustic Radiation Pressure}

John H. Cantrell

Langley Research Center, Hampton, Virginia

National Aeronautics and

Space Administration

Langley Research Center

Hampton, Virginia 23681-2199 
The use of trademarks or names of manufacturers in this report is for accurate reporting and does not constitute an official endorsement, either expressed or implied, of such products or manufacturers by the National Aeronautics and Space Administration.

Available from:

NASA STI Program / Mail Stop 148

NASA Langley Research Center

Hampton, VA 23681-2199

Fax: 757-864-6500 


\title{
Acoustic radiation pressure
}

\author{
John H. Cantrell \\ National Aeronautics and Space Administration \\ Langley Research Center \\ Nondestructive Evaluation Sciences Branch \\ Hampton, Virginia 23681, USA
}

\begin{abstract}
The theoretical foundation of acoustic radiation pressure in plane wave beams is reexamined. It is shown from finite deformation theory and the Boltzmann-Ehrenfest Adiabatic Principle that the Brillouin stress tensor (BST) is the radiation stress in Lagrangian coordinates (not Eulerian coordinates) and that the terms in the BST are not the momentum flux density and mean excess Eulerian stress but are simply contributions to the variation in the wave oscillation period resulting from changes in path length and true wave velocity, respectively, from virtual variations in the strain. It is shown that the radiation stress in Eulerian coordinates is the mean Cauchy stress (not the momentum flux density, as commonly assumed) and that Langevin's second relation does not yield an assessment of the mean Eulerian pressure, since the enthalpy used in the traditional derivations is a function of the thermodynamic tensions - not the Eulerian pressure. It is shown that the transformation between Lagrangian and Eulerian quantities cannot be obtained from the commonly-used expansion of one of the quantities in terms of the particle displacement, since the expansion provides only the difference between the value of the quantity at two different points in Cartesian space separated by the displacement. The proper transformation is obtained only by employing the transformation coefficients of finite deformation theory, which are defined in terms of the displacement gradients. Finite deformation theory leads to the result that for laterally unconfined, plane waves the Lagrangian and Eulerian radiation pressures are equal with the value $(1 / 4)\langle 2 K\rangle$ along the direction of wave propagation, where $\langle K\rangle$ is the mean kinetic energy density, and zero in directions normal to the propagation direction. This is
\end{abstract}


contrary to the Langevin result that the Lagrangian radiation pressure in the propagation direction is equal to $\langle 2 K\rangle$ and the BST result that the Eulerian radiation pressure in that direction is the momentum flux density.

Key Words: Acoustic radiation pressure, laterally confined and unconfined waves, finite deformation theory, adiabatic invariance

\section{Introduction}

The radiation pressure generated by an acoustic wave is used in a variety of applications such as acoustic radiation force-based elasticity imaging [1-9], acoustophoretic drug delivery [10], acoustic tweezers [11-15], the characterization of atomic force microscope cantilevers [16,17], and the calibration of ultrasonic transducers [18-22]. The search for the proper understanding of radiation stress in acoustic fields has been controversial and elusive since the pioneering efforts of Lord Rayleigh [23], Brillouin [24,25], Hertz and Mende [26], and Langevin [27,28] in the early twentieth century and has continued to the present time [29-52]. As pointed out by Beyer [47], the controversy is fueled by confusion arising from differing definitions, faulty assumptions, and simplifying idealizations among other factors. The focus of the present work is to understand Lagrangian and Eulerian radiation stresses (pressures) in a progressive, acoustic, plane wave beam via a direct application of finite deformation theory [53-60]. It is shown that much of the confusion results (a) from a widespread misunderstanding of Lagrangian and Eulerian coordinates and of the transformation between Lagrangian and Eulerian quantities, (b) from a misinterpretation by Brillouin of terms leading to the Brillouin stress tensor, and (c) from the long-standing assumption that the pressure defined by the enthalpy in deriving Langevin's second relation is the Eulerian pressure rather than the thermodynamic tensions (second Piola-Kirchhoff stress).

A critical analysis of Lagrangian and Eulerian coordinates and quantities is presented in Section 2 from the perspective of finite deformation theory. It is shown that the traditionally used transformation between Eulerian and Lagrangian quantities, obtained from power series expansions of either the Eulerian or Lagrangian quantity in terms of the particle displacement, is incorrect. It is shown in conjunction with Appendix A that, 
contrary to a prevalent assumption, the Lagrangian and Eulerian mass densities are equal and that, in conjunction with Appendix B, the Lagrangian and Eulerian pressures are equal in the direction of plane wave propagation - again contrary to derivations based on the traditionally used power series transformation between Eulerian and Lagrangian quantities.

A particular focus of the present work is on acoustic radiation pressure in fluids. It has long been assumed that radiation pressure in fluids is highly dependent on whether motion of fluid normal to the wave propagation direction is allowed - i.e., on whether the acoustic beam is laterally confined or laterally unconfined. Section 3 focuses on acoustic radiation pressure for laterally confined, plane wave propagation. Two independent approaches are used to assess the radiation pressure. One approach is a straightforward application of finite deformation theory. The second approach employs the BoltzmannEhrenfest Principle of Adiabatic Invariance - the approach used by Brillouin [24,25]. Both approaches lead to the result that the Brillouin stress tensor is the radiation stress referred to Lagrangian coordinates, contrary to Brillouin's assumption that the stress tensor refers to Eulerian coordinates. The radiation stress in Eulerian coordinates is shown to be the time-averaged Cauchy stress - not the Brillouin stress tensor, as traditionally assumed. Along the plane wave propagation direction, the Lagrangian and Eulerian radiation pressures are shown to be exactly equal.

Section 4 focuses on the acoustic radiation pressure for laterally unconfined plane wave beams. Again, two independent approaches are used to assess the radiation pressure - one is a direct application of finite deformation theory and the second employs the Boltzmann-Ehrenfest Adiabatic Principle. The derivations show that for both Lagrangian and Eulerian coordinates the radiation pressure along the direction of plane wave propagation is equal to $(1 / 4)\langle 2 K\rangle$, where $\langle K\rangle$ is the mean kinetic energy density of the plane wave. This result is contrary to the value $\langle 2 K\rangle$ for the Lagrangian radiation pressure obtained from the Langevin theory [27]. In directions normal to the propagation direction, the present derivations show that the radiation pressure is zero for both Lagrangian and Eulerian coordinates.

It is generally assumed that Langevin's second relation [28] refers to Eulerian coordinates and provides an assessment of the mean Eulerian excess pressure. Brillouin assumed (incorrectly, as shown in Section 3.2.2) that one of the two terms in the Brillouin 
stress tensor is the mean Eulerian excess stress (pressure). He assumed that the mean Eulerian excess stress is zero for plane wave propagation, leaving the acoustic radiation stress to result from the second term in the Brillouin stress tensor - the momentum flux density (also shown in Section 3.2.2 to be incorrect). Section 4.2 provides a critical analysis of Langevin's second relation. It is shown that the pressure associated with the enthalpy in deriving Langevin's second relation is the thermodynamic pressure (second Piola-Kirchhoff pressure) rather than the Eulerian pressure, as generally assumed, and, when accounted for, renders the Langevin's second relation invalid.

\section{Elements of finite deformation theory}

Much of the confusion surrounding acoustic radiation pressure in fluids is associated with a misunderstanding of Lagrangian and Eulerian coordinates and of the relationships between Lagrangian and Eulerian quantities. It is thus instructive to derive the relevant relationships in some detail from the perspective of finite deformation theory. The relationships between Lagrangian and Eulerian quantities are central to the theory of finite deformations, which was originally developed by Murnaghan [53], codified as a field theory by Truesdell and Toupin [58], Truesdell and Noll [59], and applied to acoustic wave propagation by Truesdell [54], Thurston [55], Thurston and Brugger [56], Thurston and Shapiro [57], and Wallace [60]. Finite deformation theory applies to any material of arbitrary crystalline symmetry including ideal fluids, which can be viewed as an isotropic material with zero shear modulus.

\subsection{Lagrangian and Eulerian coordinates}

Consider a material for which the initial (rest) configuration of particles comprising the material body is denoted by the set of position vectors $\{\boldsymbol{X}\}=\left\{X_{1}, X_{2}, X_{3}\right\}$ in a threedimensional Cartesian reference frame having unit vectors $\mathbf{e}_{1}, \mathbf{e}_{2}$, and $\mathbf{e}_{3}$ along the coordinate axes. The $\left(X_{1}, X_{2}, X_{3}\right)$ coordinates are known as Lagrangian or material (initial or rest) coordinates. Under an impressed stress the positions of the material particles will move from the initial (rest) set of vectors $\{\boldsymbol{X}\}$ to new positions described by the set of position vectors $\{\boldsymbol{x}\}=\left\{x_{1}, x_{2}, x_{3}\right\}$ in the same three-dimensional Cartesian reference frame. The $\left(x_{1}, x_{2}, x_{3}\right)$ coordinates are known as Eulerian or spatial (present) coordinates in the 
Cartesian reference frame. It is assumed that $\boldsymbol{x}$ and $\boldsymbol{X}$ are functionally related as $\boldsymbol{x}=$ $\boldsymbol{x}(\boldsymbol{X}, t)$ where $t$ is time. The present configuration of particles $\{\boldsymbol{x}\}$ is then related to the initial configuration $\{\boldsymbol{X}\}$ by means of an elastic deformation defined by the set of transformation (deformation) coefficients $\alpha_{i j}=\partial x_{i} / \partial X_{j}$, where $x_{\mathrm{i}}$ and $X_{\mathrm{j}}$, respectively, are the Cartesian components of the vectors $\boldsymbol{x}$ and $\boldsymbol{X}$. The indices $\mathrm{i}$ and $\mathrm{j}$ take the values 1,2, 3 representing the three mutually orthogonal Cartesian axes. An elemental length $d \boldsymbol{X}$ in the Lagrangian coordinates is transformed to an elemental length $d \boldsymbol{x}$ in the Eulerian coordinates as $d x_{i}=\alpha_{i j} d X_{j}$. The Einstein convention of summation over repeated indices is used in the present work. The inverse deformation is described by the set of transformation coefficients $\gamma_{i j}$ defined such that $\gamma_{i j} \alpha_{j k}=\delta_{i k}$, where $\delta_{\mathrm{ij}}$ is the Kronecker delta. If the deformation is non-uniform (i.e., varies with spatial position), the deformation is considered to be local in $\boldsymbol{X}$ and time $t$.

The deformation is defined by following the motion of a given particle originally at rest in the Lagrangian position $\boldsymbol{X}$, which during deformation is displaced to the Eulerian position $\boldsymbol{x}$. The particle displacement $\boldsymbol{u}$ is defined by $\boldsymbol{u}=\boldsymbol{x}-\boldsymbol{X}$. The transformation coefficients $\alpha_{\mathrm{ij}}$ are related to the displacement gradients $u_{i j}=\partial u_{i} / \partial X_{j}$ as

$$
\alpha_{i j}=\delta_{i j}+u_{i j}
$$

For finite deformations Murnaghan [53] pointed out that the Lagrangian strains $\eta_{\mathrm{ij}}$ defined as

$$
\eta_{i j}=\frac{1}{2}\left(\alpha_{k i} \alpha_{k j}-\delta_{i j}\right)=\frac{1}{2}\left(u_{i j}+u_{j i}+u_{k i} u_{k j}\right)
$$

are rotationally invariant and provide an alternative to the displacement gradients $u_{i j}$ as a strain measure. Eqs.(1) and (2) hold for any material system having arbitrary crystalline symmetry - solid or fluid.

\subsection{Lagrangian and Eulerian quantities}

A physical quantity $q$ in the deformed state but referred to the Lagrangian (initial, rest, or un-deformed state) coordinates at time $t$ is defined as the Lagrangian quantity 
$q^{L}(\boldsymbol{X}, t)$. The same quantity referred to the Eulerian (present or deformed state) coordinates at the same time $t$ is defined as the Eulerian quantity $q^{E}(\boldsymbol{x}, t)$. Since $q^{L}(\boldsymbol{X}, t)$ and $q^{E}(\boldsymbol{x}, t)$ represent the same physical quantity $q$ in the deformed state at the same position $\boldsymbol{x}=\boldsymbol{x}(\boldsymbol{X}, t)=\boldsymbol{X}+\boldsymbol{u}(t)$ and same time $t$ in Cartesian space, the relationship between the Lagrangian and Eulerian expressions of that quantity must necessarily be [55] $q^{L}(\boldsymbol{X}, t)=\left.q^{E}(\boldsymbol{x}, t)\right|_{\boldsymbol{X}+\boldsymbol{u}(t)}$.

It has been assumed in the acoustics literature since the early twentieth century that quantities $q^{E}(\boldsymbol{x}, t)$ in Eulerian coordinates are related to quantities $q^{L}(\boldsymbol{X}, t)$ in Lagrangian coordinates via a series expansion in the displacement $u$ as (dropping subscripts and assuming longitudinal displacements along a single Cartesian axis) [24-26,32-35,38-40,42]

$$
q^{E}(x, t)=\left.q^{L}(X, t)\right|_{X=x-u}=\left.q^{L}(X, t)\right|_{X=x}-\left.\frac{\partial q^{L}}{\partial X}(X, t)\right|_{X=x} u+\cdots
$$

or, inversely, as

$$
q^{L}(X, t)=\left.q^{E}(x, t)\right|_{x=X+u}=\left.q^{E}(x, t)\right|_{x=X}+\left.\frac{\partial q^{E}}{\partial X}(x, t)\right|_{x=X} u+\cdots
$$

It is generally assumed in Eq.(4), for example, that the Lagrangian quantity is $q^{L}(X, t)$ and that the relevant Eulerian quantity is the first term $\left.q^{E}(x, t)\right|_{x=X}$ in the series expansion. This cannot be true, because the first equality already states that the Lagrangian quantity $q^{L}(X, t)$ is equal to the Eulerian quantity $\left.q^{E}(x, t)\right|_{x=X+u}$ at the same point (deformed state) in Cartesian space at all times $t$. Indeed, the Lagrangian quantity $q^{L}(X, t)$ corresponds to the value of the quantity $q^{L}$ in the deformed state at the Eulerian position $x$ at time $t$ (corresponding to $u(t) \neq 0$ ) that previously had the value $q^{L}\left(X, t_{0}\right)$ in the un-deformed state at the initial (Lagrangian) position $X$ at the initial time $t_{0}$ (corresponding to $u\left(t_{0}\right)=$ $0)$. The first equality $q^{L}(X, t)=\left.q^{E}(x, t)\right|_{x=X+u}$ in Eq.(4) means that both the Lagrangian quantity and the Eulerian quantity involve the same particle that initially is in the undeformed position $X$ at time $t_{0}$ (corresponding to $u\left(t_{0}\right)=0$ ) in Cartesian space but has moved at time $t$ from the un-deformed position $X$ to the deformed position $x(t)=X+u(t)$ in Cartesian space. 
A Eulerian quantity represents the value of a quantity associated with a particle in the present (deformed) position. The quantity $\left.q^{E}(x, t)\right|_{x=X}$ in the expansion of Eq.(4) represents the value of a quantity associated with a particle whose present position at $x=$ $X$ originates from some un-deformed position $X^{\prime}$ in Cartesian space that is generally different from $X$. That is, the particles that appear at $x=X$ and at $x=(X+u)$ in Eulerian coordinates originate from two different Lagrangian coordinate positions in Cartesian space and, hence, represent deformations (displacements) corresponding to two different particles (except when $u=0$ ). Thus, the last equality in Eq.(4) is nothing more than the statement that the difference between the values of the Eulerian quantity evaluated at two different points in Cartesian space (corresponding to the present positions of two different particles differing by a distance $u$ ) is obtained as

$$
\left.q^{E}(x)\right|_{x=X+u}-\left.q^{E}(x)\right|_{x=X}=\left.\frac{\partial q^{E}}{\partial X}(x)\right|_{x=X} u+\cdots
$$

A similar argument regarding Eq.(3) leads to the conclusion that the difference between the values of the Lagrangian quantity evaluated at two different points in Cartesian space, differing by the distance $u$, is equal to

$$
\left.q^{L}(X)\right|_{X=x-u}-\left.q^{L}(X)\right|_{X=x}=-\left.\frac{\partial q^{L}}{\partial X}(X)\right|_{X=x} u+\cdots
$$

It is concluded that the first term in the power series expansions of Eqs.(3) and (4) does not represent the quantity conjugate to the quantity on the left of the equations and that Eqs.(3) and (4) do not define the transformation between Lagrangian and Eulerian quantities. Such a transformation can only be obtained by employing the transformation coefficients $\alpha_{i j}$ and $\gamma_{i j}$, which involve the displacement gradients rather than the displacements.

In a related issue, it is often assumed in the acoustics literature for fluids that the Eulerian coordinates correspond to surfaces fixed in Cartesian space and that the Lagrangian coordinates correspond to surfaces that oscillate in space under an impressed sinusoidal wave $[26,32,39,46]$. An oscillating material surface is defined by a set of $n$ contiguous, particle displacements $\boldsymbol{u}_{n}(t)(n=1,2,3, \cdots)$ that vary sinusoidally in time $t$. 
Relative to fixed Lagrangian coordinates $\boldsymbol{X}_{n}$, the time-dependent particle displacements $\boldsymbol{u}_{n}(t)$ are defined by $\boldsymbol{u}_{n}(t)=\boldsymbol{x}_{n}(t)-\boldsymbol{X}_{n}(n=1,2,3, \cdots)$. The Eulerian coordinates $\boldsymbol{x}$ in this case are time-dependent. Relative to fixed Eulerian coordinates $\boldsymbol{x}_{n}$ the particle displacements $\boldsymbol{u}_{n}(t)$ are defined by $\boldsymbol{u}_{n}(t)=\boldsymbol{x}_{n}-\boldsymbol{X}_{n}(t)$, where it is the Lagrangian coordinates that are now dependent on time $t$. Since it is the displacements that define the motion of the surface, it is apparent that the displacements can occur with respect to either fixed Lagrangian or fixed Eulerian coordinates - a consequence of the relativistic principle that for coordinate systems moving relative to each other it does not matter in regard to the relative displacement which system is regarded as moving and which is considered fixed.

If one chooses Lagrangian coordinates to represent points fixed in Cartesian space, then the value of a quantity at time $t$ corresponds to that following the motion of a single particle that has moved from position $\boldsymbol{X}$ to position $\boldsymbol{x}(t)=\boldsymbol{X}+\boldsymbol{u}(t)$ relative to the fixed Lagrangian coordinates $\boldsymbol{X}$. If one chooses Eulerian coordinates $\boldsymbol{x}$ to represent points fixed in Cartesian space, then (because of the definition of Lagrangian and Eulerian coordinates) the value of a quantity at a fixed position $\boldsymbol{x}$ at time $t$ corresponds to that following a succession of particles with increasing time $t$, each particle originating at different Lagrangian coordinate positions $\boldsymbol{X}$. It is crucially important to understand that points fixed in Cartesian space and points oscillating in Cartesian space are represented equally well in either Lagrangian or Eulerian coordinates. The failure to apply this relativistic principle to Lagrangian and Eulerian coordinates has led to substantial errors in quantifying acoustic radiation pressure.

\subsection{Mass density in Lagrangian and Eulerian coordinates}

A direct application of the transformation coefficients given in Eq.(1) for an initially un-deformed volume of material is shown in Appendix A to result in the wellknown relationship

$$
\begin{gathered}
\frac{\rho_{0}}{\rho}=\operatorname{det} \alpha_{i j} \equiv J \\
=1+u_{11}+u_{22}+u_{33}+u_{11} u_{22}+u_{11} u_{33}+u_{22} u_{33}+u_{11} u_{22} u_{33} .
\end{gathered}
$$

where $\rho_{0}$ is the mass density in the initial (un-deformed) state, $\rho$ is the mass density in the deformed state, and $J$ is the Jacobian of the transformation defined as the determinant of 
the transformation coefficients $\alpha_{\mathrm{ij}}$. It is important to recognize that $\rho_{0}$ is the mass density in the un-deformed state for both the Lagrangian and Eulerian coordinates and that $\rho$ is the mass density in the deformed state for both the Lagrangian and Eulerian coordinates. This is apparent from the first equality in Eqs.(3), which states that the mass density $\rho_{0}=$ $\rho^{E}\left(x, t_{0}\right)=\left.\rho^{L}\left(X, t_{0}\right)\right|_{X=x}$ is the mass density in the initial (un-deformed) state at time $t_{0}$, where $u\left(t_{0}\right)=0$, and that $\rho=\rho^{E}(x, t)=\left.\rho^{L}(X, t)\right|_{X=x-u}$ is the mass density in the deformed state at time $t$, where $u(t) \neq 0$. Similarly, from Eq.(4) $\rho_{0}=\rho^{L}\left(X, t_{0}\right)=$ $\left.\rho^{E}\left(x, t_{0}\right)\right|_{x=X}$, where $u\left(t_{0}\right)=0$, and $\rho=\rho^{L}(X, t)=\left.\rho^{E}(x, t)\right|_{x=X+u}$, where $u(t) \neq 0$. Thus, the first equality in both Eq.(3) and Eq.(4) states that the mass density $\rho$ has the same value at the same point and time in Cartesian space whether referred to the Lagrangian or Eulerian coordinates, since for either coordinates the mass density refers to the same state of deformation at a given point and time $t$. The expansion given by the last equality in Eqs.(3) and (4) leads (incorrectly) to quite different values for the mass density in the two coordinates, when the first term in the expansion is assumed to represent the relevant conjugate density.

It is very important to recognize that $\rho$ and $\rho_{0}$ represent the mass density in the deformed and undeformed states, respectively, in both Lagrangian and Eulerian coordinates. Although Eq.(5) provides an expression of $\rho$ as a function of the displacement gradients, which are referred to the Lagrangian coordinates, this does not mean that $\rho$ in Eq.(5) now becomes exclusively the Lagrangian mass density, as often assumed. It is still the mass density in the deformed state for both Lagrangian and Eulerian coordinates in accordance with the first equality in Eqs.(3) and (4). The expression of the deformed mass density in terms of the displacement gradients does not change the equality. The assumption that the Lagrangian and Eulerian mass densities are different has been fueled by the incorrect assumption that the expansion given by the last equality in Eqs.(3) and (4) provides an appropriate transformation between Lagrangian and Eulerian quantities, when the first term in the expansion is assumed to represent the relevant conjugate density.

\subsection{Stress in Lagrangian and Eulerian coordinates}

Stress is defined in terms of the derivative of the internal energy per unit volume with respect to the relevant strain measure, which leads to the stress-strain relationships. 
The internal energy per unit mass $U\left(\boldsymbol{x}, S_{m}\right)$ of material depends on the relative positions of the particles comprising the material and the entropy per unit mass $S_{m}$. This means that the internal energy per unit volume $\phi=\rho_{0} U\left(\boldsymbol{x}, S_{m}\right)$ can be expressed as a function of the displacement gradients $u_{\mathrm{ij}}$ or as a function of the Lagrangian strains $\eta_{\mathrm{ij}}$ as [60]

$$
\begin{gathered}
\phi=\rho_{0} U\left(\boldsymbol{X}, \eta_{i j}, S_{m}\right) \\
=\rho_{0} U\left(\boldsymbol{X}, 0, S_{m}\right)+C_{i j} \eta_{i j}+\frac{1}{2} C_{i j k l} \eta_{i j} \eta_{k l}+\frac{1}{3 !} C_{i j k l p q} \eta_{i j} \eta_{k l} \eta_{p q}+\cdots \\
=\rho_{0} U\left(\boldsymbol{X}, u_{i j}, S_{m}\right)=\rho_{0} U\left(\boldsymbol{X}, 0, S_{m}\right)+A_{i j} u_{i j}+\frac{1}{2} A_{i j k l} u_{i j} u_{k l}+\frac{1}{3 !} A_{i j k l p q} u_{i j} u_{k l} u_{p q}+\cdots
\end{gathered}
$$

where $A_{\mathrm{ij}}, A_{\mathrm{ijkl}}$, and $A_{\mathrm{ijklpq}}$, respectively, are the first, second, and third-order Huang coefficients and $C_{\mathrm{ij}}, C_{\mathrm{ijkl}}$, and $C_{\mathrm{ijklpq}}$, respectively, are the first, second, and third-order Brugger elastic constants [55,60]. Substituting Eq.(2) in Eq.(6) and comparing the coefficients of like powers of the displacement gradients yield the relations [60]

$$
\begin{gathered}
A_{i j}=C_{i j}=T_{i j}(\boldsymbol{X})=\left(T_{i j}\right)_{0}=\sigma_{i j}(\boldsymbol{X})=\left(\sigma_{i j}\right)_{0} \\
A_{i j k l}=T_{j l}(\boldsymbol{X}) \delta_{i k}+C_{i j k l} \\
A_{i j k l p q}=C_{j l p q} \delta_{i k}+C_{i j q l} \delta_{k p}+C_{j k q l} \delta_{i p}+C_{i j k l p q}
\end{gathered}
$$

The first-order constants $A_{i j}$ are the initial stresses at position $\boldsymbol{x}=\boldsymbol{X}$ in the material and are denoted in various alternative ways in Eq.(7) that will become apparent below.

A stress is a force per unit area obtained by differentiating Eq.(6) with respect to the appropriate strain measure, $\eta_{i j}$ or $u_{i j}$, and is thus a second rank tensor. It is noted that while the strain is defined with respect to the initial state of the material (i.e., with respect to the Lagrangian coordinates), the force $F_{\mathrm{i}}$ is usually defined with respect to a unit area of deformed material (i.e., with respect to the Eulerian coordinates) [55,58,59]. An exception is the thermodynamic tensions (second Piola-Kirchhoff stress) for which both the strain and the force are referred to the initial state $[55,58,59]$. The stresses most relevant to acoustic wave propagation are the Eulerian (Cauchy) stresses and Lagrangian (first Piola- 
Kirchhoff) stresses. The Eulerian or Cauchy stress $T_{\mathrm{ij}}$ is the force per unit area referred to the present configuration. It is a force per unit area for which both the force and the area are referred to the deformed state $\boldsymbol{x}$ [54-60]. The Cauchy stresses, evaluated in the present (perturbed or deformed) configuration $\boldsymbol{x}$, are defined in terms of the derivatives of the internal energy per unit volume with respect to the Lagrangian strains as [54-60]

$$
T_{i j}=J^{-1} \alpha_{i k} \alpha_{j l} \rho_{0}\left(\frac{\partial U}{\partial \eta_{k l}}\right)_{x, S_{m}} .
$$

Note that when evaluated at $\boldsymbol{x}=\boldsymbol{X}$ (the initial or un-deformed state) Eq.(10) yields $A_{i j}=$ $T_{i j}(\boldsymbol{X})$, the initial stresses.

The Lagrangian or first Piola-Kirchhoff stress $\sigma_{\mathrm{ij}}$ is a stress for which the force is referred to the deformed state $\boldsymbol{x}$ but the area is referred to the initial state $\boldsymbol{X}$ of the material [54-60]. It is shown in Appendix B that the Cauchy stresses are related to the first PiolaKirchhoff stresses as

$$
\sigma_{i k}=J \gamma_{k j} T_{j i}=\rho_{0} \alpha_{i m}\left(\frac{\partial U}{\partial \eta_{m k}}\right)_{x, S_{m}} .
$$

or, equivalently, as

$$
T_{i j}=\frac{1}{J} \alpha_{i k} \sigma_{j k}
$$

Eqs.(11) and (12) reveal that the relationship between the Cauchy (Eulerian) and first PiolaKirchhoff (Lagrangian) stresses is more complicated than that of the Lagrangian and Eulerian mass densities, given by the first equality in Eqs.(3) and(4). The complication results from the differing definitions of the Eulerian and Lagrangian stresses, in contrast to the single definition of the mass density as simply a mass per unit volume. Note that when evaluated at $\boldsymbol{x}=\boldsymbol{X}$ (the initial or un-deformed state) Eq.(11) yields $A_{i j}=T_{i j}(\boldsymbol{X})=\sigma_{i j}(\boldsymbol{X})$, the initial stress.

Brillouin [24,25] preferred to use the Boussinesq stress tensor $\mathrm{B}_{\mathrm{ij}}$, which is defined directly in terms of the derivatives of the internal energy per unit volume with respect to the displacement gradients. The Boussinesq stress tensor is related to the first PiolaKirchhoff stress tensor as 


$$
B_{i j}=\frac{\partial \phi}{\partial u_{i j}}=\rho_{0} \frac{\partial U}{\partial u_{i j}}=\rho_{0} \frac{\partial \eta_{k l}}{\partial u_{i j}} \frac{\partial U}{\partial \eta_{k l}}=\rho_{0} \alpha_{i k} \frac{\partial U}{\partial \eta_{k j}}=\sigma_{i j}
$$

From Eqs.(6) and (13)

$$
\sigma_{i j}=B_{i j}=A_{i j}+\frac{1}{2} A_{i j k l} u_{k l}+\frac{1}{3 !} A_{i j k l p q} u_{k l} u_{p q}+\cdots
$$

It is very important to note that for purely longitudinal, plane wave propagation along the Cartesian direction e1, the shear strains $\left(\partial u_{i} / \partial X_{j}\right)_{i \neq j}=\left(u_{i j}\right)_{i \neq j}=0, T_{i j} \rightarrow$ $T_{11} \quad, \quad \sigma_{i j} \rightarrow \sigma_{11} \quad, \quad \alpha_{i j} \rightarrow \alpha_{11}=1+\left(\partial u_{1} / \partial X_{1}\right)=1+u_{11} \quad, \quad$ and $\quad(1 / J)=[1+$ $\left.\left(\partial u_{1} / \partial X_{1}\right)\right]^{-1}=\left[1+u_{11}\right]^{-1}$. Hence, $(1 / J) \alpha_{11}=1$ and Eq.(12) simplifies without approximation to

$$
T_{11}=\sigma_{11}
$$

Eq.(15) states that for longitudinal, plane wave propagation the Lagrangian and Eulerian stresses are exactly equal. The equality results from the fact that the area components of the stresses transform such that the Lagrangian and Eulerian areas are equal during deformation. For fluids, the Cauchy stress component $T_{11}$ is related to the Eulerian pressure $p_{1}^{E}$ along $\mathbf{e}_{1}$ as $T_{11}=-p_{1}^{E}$ and the first Piola-Kirchhoff stress component $\sigma_{11}$ is related to the Lagrangian pressure $p_{1}^{L}$ along $\mathbf{e} 1$ as $\sigma_{11}=-p_{1}^{L}$ [53-60]. Eq.(15) can thus be re-written as

$$
T_{11}=-p_{1}^{E}=\sigma_{11}=-p_{1}^{L}
$$

Eq.(16) states that the Eulerian pressure $p_{1}^{E}$ is exactly equal to the Lagrangian pressure $p_{1}^{L}$ for longitudinal, plane wave propagation along $\mathbf{e}_{1}$ in materials. The subscript ' 1 ' in Eq.(16) denotes that the pressure corresponding to longitudinal, plane wave propagation along $\mathbf{e}_{\mathbf{1}}$ is the $\mathrm{i}=\mathrm{j}=1$ component of the second rank tensors $T_{i j}$ and $\sigma_{i j}$. More generally, substituting $J^{-1}$ from Eq.(5) and $\alpha_{\text {ik }}$ from Eq.(1) in Eq.(12) yields to second order in $u_{i j}$ 


$$
T_{i j} \approx \sigma_{j i}+\left(u_{i k}-\delta_{i k} u_{m m}\right) \sigma_{j k}
$$

The equations-of-state for fluids are generally defined as functions of pressure in terms of the mass density $\rho$. As shown in Section 2.3, $\rho$ refers to the mass density in the present state of deformation and has the same value whether referred to Lagrangian or Eulerian coordinates. The pressure, in contrast to the mass density, is generally different in Lagrangian and Eulerian coordinates, except for the case of purely longitudinal wave propagation. Since $\rho$ refers to the mass density in the present state of deformation, the pressure in the equations-of-state for fluids is quite naturally referred to Eulerian coordinates. Thus, the equations-of-state for fluids, when expressed as functions of the mass density, are Eulerian equations. For liquids, the equation-of-state is given as an expansion of the Eulerian pressure $p^{E}$ in terms of the mass density $\rho$ as [32]

$$
p^{E}=p_{0}+A\left(\frac{\rho-\rho_{0}}{\rho_{0}}\right)+\frac{1}{2} B\left(\frac{\rho-\rho_{0}}{\rho_{0}}\right)^{2}+\cdots
$$

where $p_{0}$ is the initial hydrostatic pressure and $\mathrm{A}$ and $\mathrm{B}$ are the Fox-Wallace-Beyer coefficients. For plane wave propagation along $\mathbf{e}_{1}$, where $u_{11} \neq 0$ and $u_{22}=u_{33}=0$, substituting $\rho=\rho_{0}\left(1+u_{11}\right)^{-1}$ from Eq.(5) in Eq.(18) leads to an expression of the pressure in terms of the strain measure $u_{11}$ as

$$
p_{1}^{E}=p_{1}^{L}=p_{0}-A u_{11}+\left(\frac{B}{2}+A\right) u_{11}^{2}+\cdots
$$

where the relation $p_{1}^{E}=p_{1}^{L}$ follows from Eq.(16) for plane waves.

For liquids, the relationship between the Brugger elastic coefficients $C_{i j k l} \ldots$ and the Fox-Wallace-Beyer coefficients are given as [61]

$$
\begin{gathered}
C_{1}=C_{2}=C_{3}=-p_{0} \\
C_{11}=C_{22}=C_{33}=A+p_{0} \\
C_{12}=C_{21}=C_{13}=C_{31}=C_{23}=C_{32}=A-p_{0} \\
C_{111}=C_{222}=C_{333}=-\left(B+5 A+3 p_{0}\right)
\end{gathered}
$$




$$
\begin{aligned}
& C_{112}=C_{121}=C_{211}=C_{113}=C_{131}=\cdots=-\left(B+A-p_{0}\right) \\
& C_{123}=C_{132}=C_{213}=C_{231}=C_{312}=C_{321}=A-B-p_{0}
\end{aligned}
$$

where $p_{0}$ is the initial (hydrostatic) pressure and the Voigt contraction of indices $(11 \rightarrow 1$, $22 \rightarrow 2,33 \rightarrow 3,23=32 \rightarrow 4,13=31 \rightarrow 5,12=21 \rightarrow 6$ ) has been used in Eqs.(20)-(25) for the Brugger coefficients. The Huang elastic coefficients $A_{i j k l \ldots}$ are assessed from Eqs.(7)-(9),(20)-(25) in terms of the Fox-Wallace-Beyer coefficients as (using Voigt contraction of indices)

$$
\begin{gathered}
A_{1}=A_{2}=A_{3}=-p_{0} \\
A_{11}=A_{22}=A_{33}=A \\
A_{12}=A_{21}=A_{13}=A_{31}=A_{23}=A_{32}=A-p_{0} \\
A_{111}=A_{222}=A_{333}=-B-2 A \\
A_{112}=A_{121}=A_{211}=A_{113}=A_{131}=\cdots=-B \\
A_{123}=A_{132}=A_{213}=A_{231}=A_{312}=A_{321}=A-B-p_{0} .
\end{gathered}
$$

The equation-of-state for ideal gases is given as $p^{E}=p_{0}\left(\rho / \rho_{0}\right)^{\gamma}$, where $\gamma$ is the ratio of specific heats. The relationships between the Huang coefficients and the corresponding elastic parameters for ideal gases are obtained by setting $A=p_{0} \gamma$ and $B=$ $p_{0} \gamma(\gamma-1)$ in Eqs.(27)-(31).

\subsection{Time-averaging of Lagrangian and Eulerian quantities}

Since acoustic radiation pressure is a time-averaged, steady-state property of the wave, it is useful to define the time-average of a continuous periodic function $f(t)$ under steady-state conditions by the operation

$$
\langle f(t)\rangle=\lim _{t \rightarrow \infty} \frac{1}{t} \int_{0}^{t} f\left(t^{\prime}\right) d t^{\prime}
$$

where the angular bracket denotes time-averaging of the function enclosed in the bracket. Note that time-averaging the Lagrangian quantity $\left\langle q^{L}(X, t)\right\rangle$ occurs while holding the 
Lagrangian coordinate fixed and time-averaging the Eulerian quantity $\left\langle q^{E}(x, t)\right\rangle$ occurs while holding the Eulerian coordinate constant. For a fixed Lagrangian coordinate $X$, the first equality in Eq.(4) yields $\left\langle q^{L}(X, t)\right\rangle=\left\langle\left. q^{E}(x, t)\right|_{x(t)=X+u(t)}\right\rangle=\left\langle q^{E}(X, t)\right\rangle$, where $\left\langle q^{E}(X, t)\right\rangle$ results from the fact that for sinusoidal waves $u(t)$ averages to zero and $x(t)$ averages to $X$. For a fixed Eulerian coordinate $x$, the first equality in Eq.(3) yields $\left\langle q^{E}(x, t)\right\rangle=\left\langle\left. q^{L}(X, t)\right|_{X(t)=x-u(t)}\right\rangle=\left\langle q^{L}(x, t)\right\rangle$, where $\left\langle q^{L}(x, t)\right\rangle$ results from the fact that for sinusoidal waves $u(t)$ averages to zero and $X(t)$ averages to $x$. When $x$ and $X$ correspond to the same point $Y$ in Cartesian space, then $x=X=Y$ and $\left\langle q^{L}(Y, t)\right\rangle=$ $\left\langle q^{E}(Y, t)\right\rangle$.

It is noted that the quantity $q$ in Eqs.(3) and (4) is assumed to be a scalar quantity defined by a single definition. The relationship between the time-averaged Cauchy (Eulerian) stress and the first Piola-Kirchhoff (Lagrangian) stress is more complicated, since stress is not a scalar but, rather, a second rank tensor, defined as a force per unit area for which the area is defined differently for the two stresses. The force in the definition of both stresses refers to Eulerian coordinates (present or deformed state) but the area in the first Piola-Kirchhoff stress refers to Lagrangian coordinates (initial or un-deformed state) and the area in the Cauchy stress refers to Eulerian coordinates. The relationship between the two stresses is thus governed by the transformation between the Lagrangian and Eulerian areas and the time-averaging must be assessed from the equation, obtained from finite deformation theory, linking the quantities. For plane, longitudinal acoustic stresses such that $\sigma_{11}=T_{11}$ at a given point in Cartesian space, $\left\langle\sigma_{11}\right\rangle=\left\langle T_{11}\right\rangle$, exactly, resulting from the fact that the areas in the two stresses transform such that the areas are equal in magnitude. In other cases, Eqs.(12) or (17) must be used in assessing the time-averaged relationship between Eulerian and Lagrangian stresses.

Finally, it is noted that since $\sigma_{\mathrm{ij}}$ is the force per unit area referred to the Lagrangian coordinates, $\left\langle\sigma_{i j}\right\rangle$ is the Lagrangian radiation stress (also known as the first Piola-Kirchhoff radiation stress). Since $T_{\mathrm{ij}}$ is the force per unit area referred to the Eulerian coordinates, $\left\langle T_{i j}\right\rangle$ is quite properly the Eulerian radiation stress (or Cauchy radiation stress). It is generally assumed in the acoustics literature that for plane wave propagation the radiation stress in Eulerian coordinates is not $\left\langle T_{i j}\right\rangle$ but, rather, the momentum flux density $\left\langle\rho v_{i} v_{j}\right\rangle$. 
It is shown in Section 3.2.2 that this assumption is based on an incorrect interpretation, originally proposed by Brillouin [24,25], of the terms in what is now known as the Brillouin stress tensor. The identification of the momentum flux density with the Eulerian radiation stress for plane waves has also led to a considerable misunderstanding of acoustic radiation stress in the literature.

\section{Acoustic radiation pressure for laterally confined, plane waves}

It has been assumed since the work of Hertz and Mende [26] that for fluids the radiation pressure in an acoustic beam is highly dependent on whether motion of fluid normal to the wave propagation direction is allowed - i.e., on whether the acoustic beam is laterally confined or laterally unconfined. Brillouin [24,25], Hertz and Mende [26], and Beyer $[32,47]$ assess the radiation pressure by assuming a longitudinal, plane wave acoustic beam of cylindrical cross-section incident on a target in laterally confined and laterally unconfined volumes. Their derivations are questionable for several reasons including an incorrect assessment of the relationship between Lagrangian and Eulerian quantities based on the expansions given in the second equality of Eqs.(3) and (4). Moreover, as pointed out by Beissner [48], a beam of finite cross-section is three-dimensional and, thus, diffracted, which leads to additional issues in assessing the radiation pressure. The seminal papers [23-28,38-40] on acoustic radiation pressure, however, assume idealized onedimensional, plane wave propagation. It will be shown below that these papers are responsible for much of the confusion and misunderstanding surrounding acoustic radiation pressure. Since the ground-breaking work on acoustic radiation pressure considered only plane waves [23-28,38-40], it is appropriate to focus on derivations of the radiation pressure for plane wave propagation in inviscid fluids, beginning with laterally confined, plane wave propagation.

\subsection{Laterally confined, plane wave propagation}

It is instructive first to consider wave propagation in Lagrangian coordinates such that the relationship between the Lagrangian stresses $\sigma_{\mathrm{ij}}$ and the displacement gradients (strains) $u_{\mathrm{ij}}$ is given by Eq.(14). Lateral confinement of a longitudinal, plane wave

propagating along $\mathbf{e}_{\mathbf{1}}$ means not only that the shear displacements gradients $\left(u_{i j}\right)_{i \neq j}=0$ 
but $u_{22}=u_{33}=0$ as well. Eq.(14) thus gives the stress-strain relationships in the Cartesian directions $\mathbf{e}_{1}, \mathbf{e}_{2}$, and $\mathbf{e}$, respectively, as

$$
\begin{aligned}
& \sigma_{11}=\left(\sigma_{11}\right)_{0}+A_{11} u_{11}+\frac{1}{2} A_{111}\left(u_{11}\right)^{2}+\cdots \\
& \sigma_{22}=\left(\sigma_{22}\right)_{0}+A_{21} u_{11}+\frac{1}{2} A_{211}\left(u_{11}\right)^{2}+\cdots \\
& \sigma_{33}=\left(\sigma_{33}\right)_{0}+A_{31} u_{11}+\frac{1}{2} A_{311}\left(u_{11}\right)^{2}+\cdots
\end{aligned}
$$

where the Voigt contraction of has been used in Eqs.(33)-(35) for the Huang coefficients. Eqs.(33)-(35) hold for all materials under laterally confined conditions. For isotropic materials, $A_{21}=A_{31}$ and $A_{211}=A_{311}$. For fluids $\left(\sigma_{11}\right)_{0}=\left(\sigma_{22}\right)_{0}=\left(\sigma_{33}\right)_{0}=-p_{0}$ where $p_{0}$ is the initial (hydrostatic) pressure.

For nonlinear, longitudinal, plane wave propagation along ê the relationship between the displacement gradient $u_{11}$ in Eqs.(33)-(35) and the particle velocity $\left(\partial u_{1} / \partial t\right)$ is given by the compatibility condition [42]

$$
\frac{\partial u_{1}}{\partial X_{1}}=u_{11}=-\frac{1}{c_{0}} \frac{\partial u_{1}}{\partial t}-\frac{1}{4 c_{0}^{2}} \frac{A_{111}}{A_{11}}\left(\frac{\partial u_{1}}{\partial t}\right)^{2}
$$

Substituting Eq.(36) in Eq.(33) and time-averaging yield the Rayleigh radiation stress (mean excess stress) along $\mathbf{e} 1$ in Lagrangian coordinates as

$$
\left\langle\sigma_{11}^{\text {Rayleigh }}\right\rangle=\left\langle\sigma_{11}\right\rangle-\left(\sigma_{11}\right)_{0}=\frac{1}{4} \frac{A_{111}}{A_{11}}\left\langle\rho_{0}\left(\frac{\partial u_{1}}{\partial t}\right)^{2}\right\rangle=\frac{1}{4} \frac{A_{111}}{A_{11}}\langle 2 K\rangle
$$

where $\langle K\rangle=(1 / 2)\left\langle\rho_{0}\left(\frac{\partial u_{1}}{\partial t}\right)^{2}\right\rangle$ is the mean kinetic energy density of the propagating wave. Since from Eq.(16) $\sigma_{11}=-p_{1}^{L}=T_{11}=-p_{1}^{E}$, the Rayleigh radiation stress (mean excess stress) in Lagrangian coordinates $\left\langle\sigma_{11}^{\text {Rayleigh }}\right\rangle$ is also equal to the Rayleigh radiation stress in Eulerian coordinates $\left\langle T_{11}^{\text {Rayleigh }}\right\rangle$, i.e. 


$$
\left\langle\sigma_{11}^{\text {Rayleigh }}\right\rangle=\left\langle T_{11}^{\text {Rayleigh }}\right\rangle=\left\langle T_{11}\right\rangle-\left(T_{11}\right)_{0}=\frac{1}{4} \frac{A_{111}}{A_{11}}\langle 2 K\rangle
$$

It is noted that the Rayleigh radiation pressure has been traditionally defined as [47] "the difference between the average pressure at a surface moving with the particle (the mean Lagrangian pressure $\left.\left\langle p_{1}^{L}\right\rangle\right)$ and the pressure that would have existed in the field of the same mean density at rest, $p_{0} . "$ This definition of the Rayleigh radiation pressure is misleading, since, as shown in Section 2.2, a moving surface is equally well represented in either Lagrangian or Eulerian coordinates. The assumption that moving surfaces are represented only by Lagrangian coordinates has led to considerable confusion, erroneous assumptions, and problematic arguments in assessing acoustic radiation pressure, as shown in Section 4.2.

For liquids with initial hydrostatic pressure $p_{0}=-\left(\sigma_{11}\right)_{0}=-\left(T_{11}\right)_{0}$, Eqs. $(27)$ and (29) yield $\left(A_{111} / A_{11}\right)=-[(B / A)+2]$. Eqs.(16), (37), and (38) state that the excess Eulerian pressure and the excess Lagrangian pressure are equal along the wave propagation direction e1. Thus, the Rayleigh radiation pressure in liquids is obtained as

$$
\left\langle p_{1}^{\text {Rayleigh }}\right\rangle=\left\langle p_{1}^{L}\right\rangle-p_{0}=\left\langle p_{1}^{E}\right\rangle-p_{0}=\frac{1}{4}\left(\frac{B}{A}+2\right)\langle 2 K\rangle
$$

The Rayleigh radiation pressure along ex for ideal gases can be obtained by substituting $B / A=(\gamma-1)$, where $\gamma$ is the ratio of specific heats, in Eq.(39) to obtain

$$
\left\langle p_{1}^{\text {Rayleigh }}\right\rangle=\left\langle p_{1}^{L}\right\rangle-p_{0}=\left\langle p_{1}^{E}\right\rangle-p_{0}=\frac{1}{4}(\gamma+1)\langle 2 K\rangle
$$

In the directions $\mathbf{e}_{2}$ and $\mathbf{e}_{3}$, normal to the wave propagation direction, the Lagrangian radiation stresses are assessed by substituting the compatibility condition, Eq.(36), in Eqs.(34) and (35), and time-averaging to obtain for isotropic materials with initial stresses $\left(\sigma_{22}\right)_{0}=\left(\sigma_{33}\right)_{0}$ 


$$
\left\langle\sigma_{22}\right\rangle-\left(\sigma_{22}\right)_{0}=\left\langle\sigma_{33}\right\rangle-\left(\sigma_{33}\right)_{0}=\left(\frac{1}{2} \frac{A_{211}}{A_{11}}-\frac{1}{4} \frac{A_{21} A_{111}}{A_{11}^{2}}\right)\langle 2 K\rangle
$$

For liquids, writing $\sigma_{22}=\sigma_{33}=-p_{2}^{L}=-p_{3}^{L}$ and substituting Eqs.(26)-(31) for $A_{11}, A_{21}$, $A_{111}$, and $A_{211}$ in Eq.(41) yield

$$
\left\langle p_{2}^{L}\right\rangle-p_{0}=\left\langle p_{3}^{L}\right\rangle-p_{0}=\frac{1}{4}\left(\frac{B}{A}-2\right)\langle 2 K\rangle+\frac{1}{4} \frac{p_{0}}{A}\left(\frac{B}{A}+2\right)\langle 2 K\rangle \approx \frac{1}{4}\left(\frac{B}{A}-2\right)\langle 2 K\rangle .
$$

where the last equality in Eq.(42) results for $p_{0} \ll A$, as is the usual case for liquids.

It is generally assumed in the literature that for ideal gases the Lagrangian radiation pressures can be obtained simply by substituting the relation $B / A=(\gamma-1)$ in the equations for liquids. Substituting $B / A=(\gamma-1)$ in the last equality in Eq.(42) for liquids leads to

$$
\left\langle p_{2}^{L}\right\rangle-p_{0}=\left\langle p_{3}^{L}\right\rangle-p_{0}=\frac{1}{4}(\gamma-3)\langle 2 K\rangle
$$

for the Lagrangian radiation pressures in directions $\mathbf{e} 2$ and $\mathbf{e} 3$. The substitution overlooks the contribution of the hydrostatic pressure $p_{0}$ as a multiplicative scaling factor in the equation-of-state for ideal gases, $p^{E}=p_{0}\left(\rho / \rho_{0}\right)^{\gamma}$, which renders the simple substitution $B / A=(\gamma-1)$ inadequate. It is more proper to set $A=p_{0} \gamma$ and $B=p_{0} \gamma(\gamma-1)$ in Eqs.(27)-(31) for the Huang coefficients and substitute the resulting Huang coefficients in Eq.(41) to get

$$
\left\langle p_{2}^{L}\right\rangle-p_{0}=\left\langle p_{3}^{L}\right\rangle-p_{0}=\frac{1}{4}(\gamma-3)\langle 2 K\rangle+\frac{1}{4} \frac{(\gamma+1)}{\gamma}\langle 2 K\rangle
$$

It is noted that Eq.(44) contains the term $\frac{1}{4} \frac{(\gamma+1)}{\gamma}\langle 2 K\rangle$ in addition to the traditionally derived term $\frac{1}{4}(\gamma-3)\langle 2 K\rangle$.

The excess Eulerian stresses along $\mathbf{e}_{2}$ and $\mathbf{e}_{3}$ are obtained from Eq.(17) for isotropic materials as (assuming equal initial stresses (pressures) in directions e2 and e3) 


$$
\begin{aligned}
\left\langle T_{22}\right\rangle-\left(T_{22}\right)_{0} & =\left\langle T_{33}\right\rangle-\left(T_{33}\right)_{0} \approx\left\langle\sigma_{22}\right\rangle-\left(\sigma_{22}\right)_{0}-\left\langle u_{11} \sigma_{22}\right\rangle \\
& =\left\langle\sigma_{33}\right\rangle-\left(\sigma_{33}\right)_{0}-\left\langle u_{11} \sigma_{33}\right\rangle
\end{aligned}
$$

where the initial stress relation $\left(T_{22}\right)_{0}=\left(\sigma_{22}\right)_{0}$ or $\left(T_{33}\right)_{0}=\left(\sigma_{33}\right)_{0}$ is subtracted from both sides of Eq.(17). For liquids,

$$
\left\langle u_{11} \sigma_{22}\right\rangle=\left\langle u_{11} \sigma_{33}\right\rangle \approx\left\langle\left(A-p_{0}\right) u_{11}^{2}\right\rangle \approx\langle 2 K\rangle
$$

where the last equality in Eq.(46) is obtained for $p_{0} \ll A$. Substituting Eq.(46) in Eq.(45) and writing $\sigma_{11}=-p_{1}^{L}, T_{11}=-p_{1}^{E}$ yield

$$
\left\langle p_{2}^{E}\right\rangle-p_{0}=\left\langle p_{3}^{E}\right\rangle-p_{0}=\frac{1}{4}\left(\frac{B}{A}+2\right)\langle 2 K\rangle
$$

Eq.(47) shows that, in contrast to the case for the Lagrangian radiation pressures, for $p_{0}<<$ $A$, the radiation pressures in Eulerian coordinates are essentially equal along directions $\mathbf{e} 1$, $\mathbf{e} 2$, and $\mathbf{e} 3$ for laterally confined, plane wave propagation in liquids.

For ideal gases, $\left\langle u_{11} \sigma_{22}\right\rangle=\left\langle u_{11} \sigma_{33}\right\rangle \approx\left\langle\left(p_{0} \gamma-p_{0}\right) u_{11}^{2}\right\rangle=\left(1-\gamma^{-1}\right)\langle 2 K\rangle$. Substituting this result in Eq.(45) leads to

$$
\left\langle p_{2}^{E}\right\rangle-p_{0}=\left\langle p_{3}^{E}\right\rangle-p_{0}=\frac{1}{4}(\gamma+1)\langle 2 K\rangle+\frac{1}{4}\left(\frac{\gamma-3}{\gamma}\right)\langle 2 K\rangle
$$

It is noted that Eq. (48) contains the term $\frac{1}{4} \frac{(\gamma-3)}{\gamma}\langle 2 K\rangle$ in addition to the term $\frac{1}{4}(\gamma+1)\langle 2 K\rangle$. Again, the extra term results from the presence of the initial pressure $p_{0}$ as a multiplicative scaling factor in the equation-of-state for ideal gases.

\subsection{Lateral confinement and the Boltzmann-Ehrenfest Adiabatic Principle}

Brillouin [24.25] approached the problem of acoustic radiation stress by applying the Boltzmann-Ehrenfest Principle of Adiabatic Invariance to longitudinal, plane wave propagation. Since the derivation of Brillouin has been so influential in establishing the 
foundational concepts of acoustic radiation stress, it is worthwhile to re-examine his approach in some detail. The Boltzmann-Ehrenfest Adiabatic Principle [62,63] states that if the constraints of a periodic system are allowed to vary sufficiently slowly, then the product of the mean (time-averaged, steady-state) kinetic energy $\left\langle K^{*}\right\rangle$ and the period $T$ of the system is an adiabatic invariant or constant of the motion such that the variation $\delta\left(\left\langle K^{*}\right\rangle T\right)=0$ or

$$
\delta\left\langle K^{*}\right\rangle=-\left\langle K^{*}\right\rangle \frac{\delta T}{T} .
$$

\subsubsection{Acoustic radiation stress and pressure for laterally confined, plane waves in}

\section{Lagrangian coordinates}

It is instructive to consider first the derivation in Lagrangian coordinates for laterally confined, longitudinal, plane wave propagation along e1. In Lagrangian coordinates, the virial theorem states that $[64,65]$

$$
\langle K\rangle=\frac{1}{2}\left\langle\frac{\partial \phi}{\partial u_{11}} u_{11}\right\rangle=\frac{1}{2}\left\langle\sigma_{11} u_{11}\right\rangle .
$$

where for longitudinal plane waves, the potential energy density corresponding to the excess stress $\left[\sigma_{11}-\left(\sigma_{11}\right)_{0}\right],\left(\sigma_{11}\right)_{0}=A_{1}$, is obtained from Eq.(6) by letting ( $\phi-$ $\left.A_{1} u_{11}\right) \rightarrow \phi^{\prime}$, dropping the prime on $\phi^{\prime}$, and writing

$$
\phi=\rho_{0} U(X, 0, S)+\frac{1}{2} A_{11} u_{11}^{2}+\frac{1}{6} A_{111} u_{11}^{3}+\cdots
$$

The relationship between the mean kinetic energy density and the mean internal (potential) energy density for plane waves can be established by substituting Eq.(51) in Eq.(50) to obtain a power series expansion of Eq.(50), and then solving Eq.(51) for $A_{11} u_{11}^{2}$ and iteratively substituting for $u_{11}^{2}$ in the terms of the expanded Eq.(50) to obtain

$$
\langle K\rangle=\langle\phi\rangle+\frac{1}{6} \frac{A_{111}}{A_{11}}\left\langle\phi u_{11}\right\rangle+\cdots
$$


where the constant term $\rho_{0} U(X, 0, S)$ has been dropped, since it makes no contribution to the kinetic energy. The mean total energy density $\langle E\rangle$ for nonlinear plane waves is then

$$
\langle E\rangle=\langle K\rangle+\langle\phi\rangle=2\langle K\rangle-\frac{1}{6} \frac{A_{111}}{A_{11}}\left\langle\phi u_{11}\right\rangle+\cdots
$$

where the last equality follows from Eq.(52). It is interesting to note from Eq.(53) that for nonlinear waves the total average energy density $\langle E\rangle$ is not exactly equal to $\langle 2 K\rangle$.

According to the Boltzmann-Ehrenfest Adiabatic Principle [62,63], a slow virtual variation $\delta q^{*}$ in a constraint $\mathrm{q}^{*}$ (generalized displacement) of a conservative, oscillatory system leads to a change in the system configuration that results in a change $\delta\left\langle E^{*}\right\rangle$ in the mean (time-averaged, steady-state) total energy $\left\langle E^{*}\right\rangle$ of the system. The change in the mean total energy is quantified by the product of the generalized reaction force $\mathrm{Q}^{*}$ and virtual constraint variation $\delta q^{*}$ such that $\delta\left\langle E^{*}\right\rangle=Q^{*} \delta q^{*}$. For longitudinal, acoustic plane wave propagation, the generalized reaction force in Lagrangian coordinates is the mean excess radiation stress $\left\langle\sigma_{11}-\left(\sigma_{11}\right)_{0}\right\rangle=\left\langle\sigma_{11}\right\rangle-\left(\sigma_{11}\right)_{0}$, the constraint (generalized displacement) is the displacement gradient $u_{11}$, the mean kinetic energy $\left\langle K^{*}\right\rangle$ corresponds to the mean kinetic energy density $\langle K\rangle$, and the mean total energy $\left\langle E^{*}\right\rangle$ corresponds to the mean total energy density $\langle E\rangle$. Thus, for plane wave propagation the relation $\delta\left\langle E^{*}\right\rangle=$ $Q^{*} \delta q^{*}$ becomes

$$
\left(\left\langle\sigma_{11}\right\rangle-\left(\sigma_{11}\right)_{0}\right) \delta u_{11}=\delta\langle E\rangle=\delta\langle 2 K\rangle-\frac{1}{6} \frac{A_{111}}{A_{11}} \delta\left\langle\phi u_{11}\right\rangle+\cdots
$$

where the last equality in Eq.(54) follows from Eq.(53).

Writing $2\left\langle K^{*}\right\rangle \rightarrow 2\langle K\rangle$ in Eq.(49) and substituting in Eq.(54) lead, to first order in the nonlinearity, to the relation for the acoustic radiation stress

$$
\left\langle\sigma_{11}\right\rangle-\left(\sigma_{11}\right)_{0}=-\langle 2 K\rangle\left(\frac{1}{T} \frac{\delta T}{\delta u_{11}}\right)_{0}-\frac{1}{6} \frac{A_{111}}{A_{11}} \frac{\delta\left\langle\phi u_{11}\right\rangle}{\delta u_{11}}
$$




$$
=-\langle 2 K\rangle\left(\frac{1}{T} \frac{\delta T}{\delta u_{11}}\right)_{0}-\frac{1}{4} \frac{A_{111}}{A_{11}}\langle 2 K\rangle
$$

where the subscripted ' 0 ' denotes evaluation at $u_{11}=0$. The factor $\frac{\delta\left\langle\phi u_{11}\right\rangle}{\delta u_{11}}$ in Eq.(55) is evaluated as

$$
\frac{\delta\left\langle\phi u_{11}\right\rangle}{\delta u_{11}}=\langle\phi\rangle+\left\langle\frac{\partial \phi}{\partial u_{11}} u_{11}\right\rangle \approx \frac{3}{2}\langle 2 K\rangle,
$$

where the last equality in Eq.(56) is a linear approximation. The linear approximation in Eq.(56) is sufficient here, since the factor containing $\frac{\delta\left\langle\phi u_{11}\right\rangle}{\delta u_{11}}$ in Eq.(55) is first order in the nonlinearity - the order retained in Eq.(55). It is noted that Brillouin omitted in his derivation the nonlinear contribution corresponding to the last term in Eq.(55).

It is extremely important to note that the radiation stress given by Eq.(55) is the Lagrangian radiation stress. The fractional change in the oscillation period $T^{-1} \delta T / \delta u_{11}$ with respect to the variation $\delta u_{11}$ can be easily assessed from the fractional change in the natural velocity $W$. The natural velocity is the velocity defined as the ratio of the length of the sound path in the un-deformed state to the propagation time in the deformed state [55-57]. Since the path length in the un-deformed state is constant, only the propagation time in the deformed state plays a role in assessing the fractional variation in the system period when using the natural velocity for the assessment. The natural velocity is the velocity referred to the Lagrangian coordinates and is obtained from Eq.(14) as [55-57]

$$
W^{2}=\frac{1}{\rho_{0}} \frac{\partial \sigma_{11}}{\partial u_{11}}=\frac{1}{\rho_{0}}\left(A_{11}+A_{111} u_{11}+\cdots\right)
$$

The fractional change in the period $\left(T^{-1} \delta T / \delta u_{11}\right)_{0}$ is assessed from the fractional change in the natural velocity as

$$
\left(\frac{1}{T} \frac{\delta T}{\delta u_{11}}\right)_{0}=-\left(\frac{1}{W} \frac{\delta W}{\delta u_{11}}\right)_{0}=-\frac{1}{2} \frac{A_{111}}{A_{11}}
$$


The acoustic radiation stress in Lagrangian coordinates is evaluated from Eqs.(55), (56), and (58) as

$$
\left\langle\sigma_{11}^{\text {Rayleigh }}\right\rangle=\left\langle\sigma_{11}\right\rangle-\left(\sigma_{11}\right)_{0}=\frac{1}{4} \frac{A_{111}}{A_{11}}\langle 2 K\rangle
$$

Eq.(59) is identical to Eq.(37) obtained from finite deformation theory.

For liquids with initial (hydrostatic) pressure $p_{0}$, Eq.(16) yields that the excess Eulerian pressure and the excess Lagrangian radiation pressure are equal along the wave propagation direction $\mathbf{e}_{1}$ and Eqs.(27) and (29) yield $\left(A_{111} / A_{11}\right)=-[(B / A)+2]$. Thus, for liquids Eq.(59) gives

$$
\left\langle p_{1}^{\text {Rayleigh }}\right\rangle=\left\langle p_{1}^{L}\right\rangle-p_{0}=\left\langle p_{1}^{E}\right\rangle-p_{0}=\frac{1}{4}\left(\frac{B}{A}+2\right)\langle 2 K\rangle
$$

in agreement with Eq.(39), obtained from finite deformation theory. For ideal gases, substituting $B / A=(\gamma-1)$ in Eq.(60) leads to

$$
\left\langle p_{1}^{\text {Rayleigh }}\right\rangle=\left\langle p_{1}^{L}\right\rangle-p_{0}=\left\langle p_{1}^{E}\right\rangle-p_{0}=\frac{1}{4}(\gamma+1)\langle 2 K\rangle
$$

in agreement with Eq.(40), obtained from finite deformation theory.

In directions $\mathbf{e}_{2}$ and $\mathbf{e}_{3}$, the derivation of the radiation pressure from the BoltzmannEhrenfest Adiabatic Principle is less straightforward and deviates somewhat from the conditions under which the Principle is strictly applicable, which in the present case requires a slow, virtual variation in the displacement gradient $u_{11}$, serving as the constraint parameter (generalized displacement) directly affecting the system period. It is, nonetheless, instructive to apply the Principle to an assessment of the radiation pressure along $\mathbf{e}_{2}$ and $\mathbf{e}_{3}$. The lateral confinement condition, $u_{22}=u_{33}=0$, means that although a variation in $u_{11}$ must lead to variations in $\sigma_{22}$ and $\sigma_{33}$ in accordance with Eqs.(34) and (35), no sound wave actually propagates in directions $\mathbf{e}_{2}$ and $\mathbf{e}_{3}$ and no constraint parameter (generalized displacement) along those directions plays a dynamically active role in affecting the system period. However, the variations of $\sigma_{22}$ and $\sigma_{33}$ occur in a confined volume in which the only allowed volume change results from variations in $u_{11}$. The 
confinement necessarily leads to a change in the natural sound velocities along directions $\mathbf{e}_{2}$ and $\mathbf{e}_{3}$. In analogy to the natural velocity $W$ along $\mathbf{e}_{1}$, which is defined as $W^{2}=$ $\left(\partial \sigma_{11} / \partial u_{11}\right)_{u_{11}=0}=\left(A_{11} / \rho_{0}\right)$, it is appropriate to define the natural velocities $W_{2}$ and $W_{3}$ along directions $\mathbf{e}_{\mathbf{2}}$ and $\mathbf{e}_{3}$, respectively, for isotropic materials as

$$
W_{2}^{2}=W_{3}^{2}=\left(\partial \sigma_{22} / \partial u_{11}\right)_{u_{11}=0}=\left(\partial \sigma_{33} / \partial u_{11}\right)_{u_{11}=0}=\left(A_{21} / \rho_{0}\right)=\left(A_{31} / \rho_{0}\right)
$$

The elastic coefficients $A_{21}$ and $A_{31}$ serve to connect the driving dynamics along e1 with dynamics along $\mathbf{e} 2$ and $\mathbf{e} 3$.

For isotropic materials, the Boltzmann-Ehrenfest equation along $\mathbf{e}_{2}$ and $\mathbf{e}_{3}$ is correspondingly written as

$$
\begin{aligned}
\left\langle\sigma_{22}\right\rangle-\left(\sigma_{22}\right)_{0}=\left\langle\sigma_{33}\right\rangle & -\left(\sigma_{33}\right)_{0}=\langle 2 K\rangle\left(\frac{1}{W_{2}} \frac{\delta W_{2}}{\delta u_{11}}\right)_{0}-\frac{1}{6} \frac{A_{111}}{A_{11}} \frac{\delta\left\langle\phi u_{11}\right\rangle}{\delta u_{11}} \\
= & \left(\frac{1}{2} \frac{A_{211}}{A_{21}}-\frac{1}{4} \frac{A_{111}}{A_{11}}\right)\langle 2 K\rangle
\end{aligned}
$$

where the nonlinear contribution from the driving wave along $\mathbf{e}_{\mathbf{1}}$ is again given as $\frac{1}{6} \frac{A_{111}}{A_{11}} \frac{\delta\left\langle\phi u_{11}\right\rangle}{\delta u_{11}}$. As anticipated, Eq.(63) is quite similar but not identical to Eq.(41). However, for liquids such that $p_{0} \ll A$, Eq.(63) and Eqs.(26)-(31) yield the Lagrangian radiation pressure along $\mathbf{e}_{2}$ and $\mathbf{e}_{3}$ as

$$
\left\langle p_{2}^{L}\right\rangle-p_{0}=\left\langle p_{3}^{L}\right\rangle-p_{0}=\frac{1}{4}\left(\frac{B}{A}-2\right)\langle 2 K\rangle
$$

Eq.(64) is in agreement with Eq.(42), obtained from finite deformation theory. For ideal gases, substituting Eqs.(26)-(31) and the relations $A=p_{0} \gamma$ and $B=p_{0} \gamma(\gamma-1)$ in Eq.(63) leads to

$$
\left\langle p_{2}^{L}\right\rangle-p_{0}=\left\langle p_{3}^{L}\right\rangle-p_{0}=\frac{1}{4}(\gamma-3)\langle 2 K\rangle+\frac{1}{2}\langle 2 K\rangle
$$

The first terms on the right-hand sides of Eqs.(65) and (44) are identical. However, the 
term $\frac{1}{2}\langle 2 K\rangle$ in Eq.(65) does not agree with the term $\frac{1}{4} \frac{(\gamma+1)}{\gamma}\langle 2 K\rangle$ obtained in Eq.(44). This results from the difference between Eqs.(41) and (63). As stated above, the derivation of the radiation pressure in directions $\mathbf{e} 2$ and $\mathbf{e} 3$ from the Boltzmann-Ehrenfest Adiabatic Principle deviates from the conditions under which the Principle is strictly applicable. Thus, the results of Eq.(65) are not completely consistent with the results of finite deformation theory and must be taken with caution, giving deference to Eq.(44).

It is noted that if the relation $B / A=(\gamma-1)$ is substituted directly in Eq.(64), Eq.(43) is obtained. This is the result obtained by Brillouin in his application of the BoltzmannEhrenfest Adiabatic Principle. Eqs.(60) and (61), Eq.(64), and Eq.(43) played a significant role in the early theoretical development of acoustic radiation pressure, since the results given on the right-hand side of the equations are exactly the results obtained by Brillouin (after correcting for the last term in Eq.(55), which was omitted by Brillouin in his derivation). However, Brillouin incorrectly assumed that the equations refer to Eulerian coordinates rather than to Lagrangian coordinates, as shown in the present derivations. It is crucial to understand how Brillouin came to such an assumption, since the assumption has led to a deep foundational misunderstanding of acoustic radiation stress and pressure.

\subsubsection{Acoustic Radiation stress and pressure in Lagrangian coordinates via the true velocity}

The true velocity $c$ is the velocity defined as the ratio of the length of the sound path in the deformed state to the propagation time in the deformed state. The true velocity is related to the natural velocity $W$ as $W=\frac{\ell_{0}}{\ell} c$, where $\ell_{0}$ is the length of material in the un-deformed state and $\ell$ is the length of material in the deformed state [52-54,57]. It is shown in Appendix B, Eq.(B6), that the ratio $R=\frac{\ell_{0}}{\ell}$ is obtained as $R=\frac{\ell_{0}}{\ell}=$ $\left[\left(\delta_{i j}-2 \frac{\partial u_{i}}{\partial X_{j}}\right) N_{i} N_{j}\right]^{1 / 2}$, where $N_{\mathrm{i}}$ are the Cartesian components of the unit vector normal to the material surface in the un-deformed (initial) state. Thus, the true velocity and the natural velocity are related as 


$$
W=\frac{\ell_{0}}{\ell} c=\left[\left(\delta_{i j}-2 \frac{\partial u_{i}}{\partial X_{j}}\right) N_{i} N_{j}\right]^{1 / 2} c
$$

In terms of variations in the true velocity $\left(\frac{1}{c} \frac{\delta c}{\delta u_{11}}\right)_{0}$ the variation of the system period $T$ is obtained from Eqs.(58) and (66) as

$$
\left(\frac{1}{T} \frac{\delta T}{\delta u_{11}}\right)_{0}=-\left(\frac{1}{W} \frac{\delta W}{\delta u_{11}}\right)_{0}=-\left(\frac{1}{c} \frac{\delta c}{\delta u_{11}}\right)_{0}-\left(\frac{1}{R} \frac{\delta R}{\delta u_{11}}\right)_{0}=-\left(\frac{1}{c} \frac{\delta c}{\delta u_{11}}\right)_{0}+1=-\frac{1}{2} \frac{A_{111}}{A_{11}}
$$

where $\left[R^{-1}\left(\delta R / \delta u_{11}\right)\right]_{0}=-1$. It is significant to point out that the terms in Eq.(67) are evaluated at $u_{11}=0$, i.e., at the Lagrangian coordinates $\boldsymbol{X}$.

The Lagrangian radiation stress for laterally confined, longitudinal, plane waves along $\mathbf{e}_{1}$ can be assessed in terms of the true velocity by substituting Eq.(67) in Eq.(55) to obtain

$$
\begin{gathered}
\left\langle\sigma_{11}\right\rangle-\left(\sigma_{11}\right)_{0}=\langle 2 K\rangle\left(\frac{1}{W} \frac{\delta W}{\delta u_{11}}\right)_{0}-\frac{1}{4} \frac{A_{111}}{A_{11}}\langle 2 K\rangle \\
=\langle 2 K\rangle\left(\frac{1}{c} \frac{\delta c}{\delta u_{11}}-1\right)_{0}-\frac{1}{4} \frac{A_{111}}{A_{11}}\langle 2 K\rangle .
\end{gathered}
$$

It is very important to recognize that Eq.(68) is an equation in Lagrangian coordinates. Although the path length for the true velocity $c$ refers to the deformed state, the term $\left(\frac{1}{c} \frac{\delta c}{\delta u_{11}}-1\right)_{0}$ in the last equality in Eq.(68) is evaluated at the un-deformed state, i.e., at the Lagrangian coordinate position $\boldsymbol{X}$, where $u_{11}=0$. Brillouin [24,25] identified the term $\langle 2 K\rangle=-\langle 2 K\rangle\left(\frac{1}{R} \frac{\delta R}{\delta u_{11}}\right)_{0}$ in Eq.(68) as the momentum flux density $\left\langle\rho v_{1} v_{1}\right\rangle$ and identified the term $\langle 2 K\rangle\left(\frac{1}{c} \frac{\delta c}{\delta u_{11}}\right)_{0}$ as the mean excess Cauchy (Eulerian) stress, which for fluids is generally called the 'mean excess Eulerian pressure.' Since $c^{2}$ is obtained for fluids from the Eulerian pressure $p^{E}$ as $c^{2}=\partial p^{E} / \partial \rho$, which is a Eulerian expression, it is, indeed, tempting to assume that $\langle 2 K\rangle\left(\frac{1}{c} \frac{\delta c}{\delta u_{11}}\right)_{0}$ corresponds to the mean excess radiation pressure in Eulerian coordinates, but the term is evaluated at $u_{11}=0$, i.e., at the Lagrangian 
coordinates $\boldsymbol{X}$. Moreover, taking the derivative of $c$ with respect to $u_{11}$ requires that $c$ be expressed in terms of $u_{11}$. Since $u_{11}$ is defined with respect to the Lagrangian coordinates, the expression of $c$ in terms of $u_{11}$ allows $c$ to be evaluated with respect to Lagrangian coordinates. This does not imply that $\mathrm{c}$ is the natural velocity; $c$ is still the true velocity but simply evaluated with respect to the Lagrangian coordinates. This means that $\langle 2 K\rangle\left(\frac{1}{c} \frac{\delta c}{\delta u_{11}}\right)_{0}$ is also referred to the Lagrangian coordinates and confirms that Eq.(68) is a Lagrangian equation.

Further, Eq.(68) shows that $\langle 2 K\rangle\left(\frac{1}{c} \frac{\delta c}{\delta u_{11}}\right)_{0}$ cannot be the mean excess Cauchy (Eulerian) stress $\left\langle T_{11}\right\rangle-\left(T_{11}\right)_{0}$, since Eq.(16) already establishes that $\left\langle T_{11}\right\rangle-\left(T_{11}\right)_{0}=$ $\left\langle\sigma_{11}\right\rangle-\left(\sigma_{11}\right)_{0}$. The term $\langle 2 K\rangle\left(\frac{1}{c} \frac{\delta c}{\delta u_{11}}\right)_{0}$ cannot be equal to both the mean excess Lagrangian stress and the mean excess Eulerian stress, since the term $\langle 2 K\rangle=$ $-\langle 2 K\rangle\left(\frac{1}{R} \frac{\delta R}{\delta u_{11}}\right)_{0}$ also appears in Eq.(68). Rather, $\langle 2 K\rangle\left(\frac{1}{c} \frac{\delta c}{\delta u_{11}}\right)_{0}$ is simply the contribution to the Lagrangian radiation stress along e1 resulting from the change in the true sound velocity, when the variation in the system period is assessed using the true velocity. The radiation stress (pressure) in Eulerian coordinates is obtained from the time-averaged Lagrangian stress (pressure) via the transformation given by Eqs.(12) or (17), which for fluids leads to Eqs.(39), (40), (47), and (48).

It is seen from Eqs.(67) and (68) that the term $\langle 2 K\rangle=-\langle 2 K\rangle\left(\frac{1}{R} \frac{\delta R}{\delta u_{11}}\right)_{0}$, which Brillouin identified as the momentum flux density, is simply the contribution to the Lagrangian radiation stress along $\mathbf{e}_{1}$ resulting from the variation in the acoustic path length in response to the virtual variation in the strain $u_{11}$, when the variation in the system period is assessed using the true velocity. The momentum flux density appears in acoustical equations in Eulerian coordinates as a consequence of the convective derivative in the Eulerian equations of motion. It does not appear in acoustical equations in Lagrangian coordinates, since the convective derivative does not appear in the Lagrangian equations of motion. Since Eq.(68) provides an assessment of the acoustic radiation stress in Lagrangian coordinates, it follows that $\langle 2 K\rangle=-\langle 2 K\rangle\left(\frac{1}{R} \frac{\delta R}{\delta u_{11}}\right)_{0}$ cannot be the momentum flux density. 
Adding to the confusion generated by the incorrect identification of the $\langle 2 K\rangle\left(\frac{1}{c} \frac{\delta c}{\delta u_{11}}\right)_{0}$ and $\langle 2 K\rangle\left(\frac{1}{R} \frac{\delta R}{\delta u_{11}}\right)_{0}$ terms in Eqs.(67) and (68), Brillouin failed to recognize that the term $\left\langle\sigma_{11}\right\rangle-\left(\sigma_{11}\right)_{0}$ in Eq.(68) is actually the Lagrangian (first Piola-Kirchhoff) stress. Brillouin identified the term $\left\langle\sigma_{11}\right\rangle-\left(\sigma_{11}\right)_{0}$ in Eq. $(68)$ as the $(i=\mathrm{j}=1)$ component of an entirely different tensor, known today as the Brillouin stress tensor $S_{11}$, which he assumed to represent the radiation stress in Eulerian coordinates. Brillouin's tensor is written more generally as $S_{i j}=\left\langle\sigma_{i j}\right\rangle-\left(\sigma_{i j}\right)_{0}$.

The improper identification of $S_{i j}$ as the radiation stress in Eulerian coordinates has also occurred from a consideration of the wave equation written in 'conservative' form as $[33,42,46]$

$$
\frac{\partial\left\langle T_{j i}-\rho v_{i} v_{j}\right\rangle}{\partial x_{j}}=\left\langle\frac{\partial\left(\rho v_{i}\right)}{\partial t}\right\rangle=0
$$

where $T_{j i}$ is the Cauchy stress, $\rho v_{i} v_{j}$ is the momentum flux density, and the last equality results from Eq.(32) and the boundedness of $\rho v_{i}$ as $t \rightarrow \infty$. For plane wave propagation along $\mathbf{e}_{1}$, integration of Eq.(69) yields $\left\langle T_{11}\right\rangle-\left(T_{11}\right)_{0}-\left\langle\rho v_{1} v_{1}\right\rangle=0$ where the constant of integration is set equal to the initial Cauchy stress $\left(T_{11}\right)_{0}$. The factor $\left\langle T_{11}\right\rangle-\left(T_{11}\right)_{0}$ is identified as the 'mean Eulerian excess stress' and the linear combination of terms, $\left\langle T_{11}\right\rangle-$ $\left(T_{11}\right)_{0}-\left\langle\rho v_{1} v_{1}\right\rangle$ is identified as the $S_{11}$ component of the Brillouin stress tensor

$$
S_{11}=\left\langle T_{11}\right\rangle-\left(T_{11}\right)_{0}-\left\langle\rho v_{1} v_{1}\right\rangle
$$

According to Eq.(68), however, $\left\langle S_{11}\right\rangle$ is actuality $\left\langle S_{11}\right\rangle=\left\langle\sigma_{11}\right\rangle-\left(\sigma_{11}\right)_{0}$ and, according to Eq.(16), $\left\langle\sigma_{11}\right\rangle-\left(\sigma_{11}\right)_{0}=\left\langle T_{11}\right\rangle-\left(T_{11}\right)_{0}$, where the initial stress $\left(\sigma_{11}\right)_{0}=\left(T_{11}\right)_{0}$. Substituting these equalities in Eq.(70) leads to the result that the momentum flux density is zero along $\mathbf{e}_{1}-$ a result consistent with the Brillouin stress tensor being a Lagrangian tensor, since the momentum flux density does not appear in the equations of motion in Lagrangian coordinates. It is again concluded that the Brillouin stress tensor does not represent the radiation stress in Eulerian coordinates, as posited by Brillouin. 


\section{Acoustic radiation pressure for laterally unconfined, plane waves}

\subsection{Laterally unconfined, plane wave propagation}

In assessing acoustic radiation pressure for laterally unconfined plane waves propagating along $\mathbf{e}_{\mathbf{1}}$ in inviscid fluids, it is crucial to recognize that the time-averaged energy density $\langle 2 K\rangle$ ( $K=$ kinetic energy density) that drives the radiation pressure is produced by a sinusoidally oscillating plane wave of finite beam cross-section (usually cylindrical) propagating under laterally constrained conditions. That is, the dynamic wave propagation is defined such that $u_{11} \neq 0, u_{22}=u_{33}=0$. In contrast, the radiation pressure itself is governed by static (time-averaged, steady-state) conditions associated with a laterally unconstrained volume. This is quite unlike the case for laterally confined, plane wave beams where both the dynamical wave and the radiation (static) pressure are subject to the same lateral constraints, $u_{11} \neq 0, u_{22}=u_{33}=0$.

Strictly, for laterally unconfined conditions a cylindrical acoustic beam of finite cross-section is not planar because of diffraction, but becomes increasingly planar in an area around the center of the beam as the ratio of the acoustic wavelength to the beam radius $r$ approaches zero. Further, as pointed out by Lee and Wang [46] the amplitude of the wave does not abruptly decrease to zero beyond the beam radius but does so smoothly in a manner approximated by the zeroth order Bessel function $J_{0}\left(\alpha_{c} r\right)$, where $\alpha_{c}$ is a constant corresponding to the reciprocal of some characteristic beam radius. For present purposes, there is no loss in generality for one-dimensional wave propagation to assume an idealized plane wave beam of cylindrical cross-section with a 'top-hat' amplitude profile. More importantly, as shown below, lateral unconfinement modifies the assessment of the elastic coefficients relevant to static conditions.

The radiation-induced static strain generated by the acoustic plane wave statically deforms the volume of material through which the wave propagates. The reaction of the statically deformed volume, however, is governed not by the dynamic, laterally constrained conditions associated with plane wave propagation but by the static (time-averaged, steady state) conditions governing a laterally unconstrained volume. To emphasize that the elastic properties associated with static, laterally unconstrained conditions are distinct from those of the dynamic, laterally constrained conditions associated with dynamic, acoustic plane wave propagation, the functions, parameters, and variables associated with static, laterally 
unconstrained conditions are designated by the superscript ' $\mathrm{S}$ '. Thus, for static, laterally unconstrained conditions $\left\langle u_{i j}\right\rangle \rightarrow\left\langle u_{i j}^{S}\right\rangle,\left\langle u_{i j} u_{k l}\right\rangle \rightarrow\left\langle u_{i j}^{S} u_{k l}^{S}\right\rangle,\left\langle\sigma_{i j}\right\rangle \rightarrow\left\langle\sigma_{i j}^{S}\right\rangle, A_{i j} \rightarrow A_{i j}^{S}$, etc.

For static, laterally unconstrained conditions in inviscid fluids, not only are the mean shear displacements gradients $\left\langle u_{i j}^{S}\right\rangle_{i \neq j}=0$ but also the mean dilatation $\left\langle\Theta^{S}\right\rangle=$ $\left\langle\Delta V^{S} / V_{0}^{S}\right\rangle=\left\langle u_{11}^{S}\right\rangle+\left\langle u_{22}^{S}\right\rangle+\left\langle u_{33}^{S}\right\rangle=0$, where $\left\langle\Delta V^{S}\right\rangle$ is the mean change in the initial volume $V_{0}^{S}$ [66]. For plane wave propagation along $\mathbf{e}_{1}$, the symmetry in directions $\mathbf{e}_{2}$ and e3 and the null mean dilatation, $\left\langle\Theta^{S}\right\rangle=0$, require that $\left\langle u_{22}^{S}\right\rangle=\left\langle u_{33}^{S}\right\rangle=-(1 / 2)\left\langle u_{11}^{S}\right\rangle$. Thus, unlike the case for laterally constrained conditions, where the strains $u_{11}, u_{22}$, and $u_{22}$ (hence, $\left\langle u_{11}\right\rangle,\left\langle u_{22}\right\rangle$, and $\left\langle u_{33}\right\rangle$ ), are independent, the relationship $\left\langle u_{22}^{S}\right\rangle=\left\langle u_{33}^{S}\right\rangle=$ $-(1 / 2)\left\langle u_{11}^{S}\right\rangle$ for static, laterally unconstrained conditions reduces the number of independent static strains to one, given as $\left\langle u_{11}^{S}\right\rangle$. The relationship $\left\langle u_{22}^{S}\right\rangle=\left\langle u_{33}^{S}\right\rangle=$ $-(1 / 2)\left\langle u_{11}^{S}\right\rangle$ implies that there is free flow of fluid in the directions $\mathbf{e} 2$ and $\mathbf{e} 3$ under static, laterally unconstrained conditions.

It is convenient to begin with a consideration of Lagrangian coordinates. Performing the summation in the last equality in Eq.(6), time-averaging, and within the time-averaging substituting Eqs.(26)-(31) for the Huang coefficients and the relation $u_{22}^{S}=$ $u_{33}^{S}=-(1 / 2) u_{11}^{S}$ corresponding to laterally unconstrained conditions lead to an assessment of the mean internal energy density $\left\langle\phi^{S}\right\rangle$ as (using Voigt contraction of indices for the elastic coefficients)

$$
\left\langle\phi^{S}\right\rangle=\rho_{0} U(X, 0, S)+A_{1}^{S}\left\langle u_{11}^{S}\right\rangle+\frac{1}{2} A_{11}^{S}\left\langle\left(u_{11}^{S}\right)^{2}\right\rangle+\frac{1}{3 !} A_{111}^{S}\left\langle\left(u_{11}^{S}\right)^{3}\right\rangle+\cdots
$$

where

$$
A_{1}^{S}=-p_{0}, \quad A_{11}^{S}=\frac{3}{2} p_{0} \quad, \quad A_{111}^{S}=-\frac{3}{2} p_{0}
$$

The $\left\langle\sigma_{11}^{S}\right\rangle$ component of the static (radiation) stress along $\mathbf{e}_{\mathbf{1}}$ is obtained as

$$
\left\langle\sigma_{11}^{S}\right\rangle=\frac{\partial\left\langle\phi^{S}\right\rangle}{\partial u_{11}^{S}}=A_{1}^{S}+A_{11}^{S}\left\langle u_{11}^{S}\right\rangle+\frac{1}{2} A_{111}^{S}\left\langle\left(u_{11}^{S}\right)^{2}\right\rangle+\cdots
$$

It is noted from Eq.(72) that the Fox-Wallace-Beyer coefficients $A$ and $B$ do not appear in 
Eqs.(71) and (73) - only the initial (hydrostatic) pressure $p_{0}$ appears. This results from the fact that, because the shear modulus for inviscid fluids is zero, the fluid freely deforms under static loads.

The static strain $\left\langle u_{11}^{S}\right\rangle$ in direction $\mathbf{e}_{1}$ can be assessed in terms of the particle velocity $\left(\partial u_{1} / \partial t\right)$ associated with the driving, dynamical, plane wave propagation from the compatibility condition, Eq.(36), as

$$
\begin{gathered}
\left\langle u_{11}^{S}\right\rangle=-\frac{1}{4\left(c_{0}^{S}\right)^{2}} \frac{A_{111}^{S}}{A_{11}^{S}}\left\langle\left(\frac{\partial u_{1}}{\partial t}\right)^{2}\right\rangle \\
\left\langle\left(u_{11}^{S}\right)^{2}\right\rangle=\frac{1}{\left(c_{0}^{S}\right)^{2}}\left\langle\left(\frac{\partial u_{1}}{\partial t}\right)^{2}\right\rangle
\end{gathered}
$$

where $c_{0}^{S}=\left(A_{11}^{S} / \rho_{0}\right)^{1 / 2}$. It is noted that the elastic constants $A_{11}^{S}$ and $A_{111}^{S}$ in Eqs.(74) and (75) reflect the static, laterally unconstrained conditions, while the particle velocity $\left(\partial u_{1} / \partial t\right)$ refers to the dynamical wave oscillations that drive the static displacement. Eqs.(74) and (75) thus establish the connection between the static strains and the mean (time-averaged) energy density of the driving dynamical wave. Substituting Eqs.(74) and (75) in Eq.(73), noting from Eq.(16) that for plane wave propagation in direction $\mathbf{e}_{1}$ the Lagrangian and Eulerian pressures are exactly equal such that $\left\langle\sigma_{11}^{S}\right\rangle=\left\langle T_{11}^{S}\right\rangle=-\left\langle p_{1}^{L}\right\rangle=$ $-\left\langle p_{1}^{E}\right\rangle$, and writing $\left\langle\rho_{0}\left(\frac{\partial u_{1}}{\partial t}\right)^{2}\right\rangle=\langle 2 K\rangle$ lead to

$$
\left\langle p_{1}^{E}\right\rangle-p_{0}=\left\langle p_{1}^{L}\right\rangle-p_{0}=\frac{1}{4}\langle 2 K\rangle
$$

Eq.(76) is quite different from the result obtained from the Langevin theory [27], which leads to the relation $\left\langle p_{1}^{L}\right\rangle-p_{0}=\langle 2 K\rangle$ for the mean excess Lagrangian pressure for laterally unconfined, plane waves.

Confirmation of Eq.(76) for propagation along $\mathbf{e} 1$ can be obtained straightforwardly from the Boltzmann-Ehrenfest Adiabatic Principle by re-writing Eq.(55) for the case of static, laterally unconstrained conditions as 


$$
\begin{gathered}
\left\langle p_{1}^{E}\right\rangle-p_{0}=\left\langle p_{1}^{L}\right\rangle-p_{0}=-\langle 2 K\rangle\left(\frac{1}{W^{S}} \frac{\delta W^{S}}{\delta u_{11}^{S}}\right)_{0}+\frac{1}{6} \frac{A_{111}^{S}}{A_{11}^{S}} \frac{\delta\left\langle\phi^{S} u_{11}^{S}\right\rangle}{\delta u_{11}^{S}} \\
=-\frac{1}{4} \frac{A_{111}^{S}}{A_{11}^{S}}\langle 2 K\rangle=\frac{1}{4}\langle 2 K\rangle
\end{gathered}
$$

where in Eq.(77) the constraint parameter q* (generalized displacement) in the BoltzmannEhrenfest Principle is now designated as $u_{11}^{S}$ to reflect that the radiation pressure is now governed by static, laterally unconstrained conditions. The natural velocity $W^{S}$ under such conditions is correspondingly defined as $\left(W^{S}\right)^{2}=\rho_{0}^{-1}\left(\partial\left\langle\sigma_{11}^{S}\right\rangle / \partial u_{11}^{S}\right)$. Thus, $\left[\left(W^{S}\right)^{-1} \delta W^{S} / \delta u_{11}^{S}\right]_{0}=(1 / 2)\left(A_{111}^{S} / A_{11}^{S}\right)=-(1 / 2)$ in Eq.(77). The term $\left(\frac{1}{6} \frac{\delta\left\langle\phi^{S} u_{11}^{S}\right\rangle}{\delta u_{11}^{S}}\right)$ in Eq.(77) is evaluated in the linear approximation, using Eq.(75), as $\frac{1}{6} \frac{\delta\left\langle\phi^{S} u_{11}^{S}\right\rangle}{\delta u_{11}^{S}}=\frac{1}{4}\langle 2 K\rangle$. The linear approximation is sufficient here, since the factor containing $\frac{\delta\left\langle\phi^{S} u_{11}^{S}\right\rangle}{\delta u_{11}^{S}}$ in Eq.(77) is first order in the nonlinearity - the order retained in Eq.(77). The relation given by Eq.(76), obtained from finite deformation theory, is thus confirmed by Eq.(77), obtained from the Boltzmann-Ehrenfest Adiabatic Principle.

The static stresses $\left\langle\sigma_{22}^{S}\right\rangle$ and $\left\langle\sigma_{33}^{S}\right\rangle$, respectively, in the directions $\mathbf{e}_{2}$ and $\mathbf{e}_{3}$ are obtained from the mean internal energy density $\left\langle\phi^{S}\right\rangle$ as $\left\langle\sigma_{22}^{S}\right\rangle=\partial\left\langle\phi^{S}\right\rangle / \partial u_{22}^{S}$ and $\left\langle\sigma_{33}^{S}\right\rangle=$ $\partial\left\langle\phi^{S}\right\rangle / \partial u_{33}^{S}$. However, because of the symmetry in the directions $\mathbf{e}_{2}$ and $\mathbf{e}_{3}$ and the dilatation relationship $\left\langle\Theta^{S}\right\rangle=\left\langle\Delta V^{S} / V_{0}^{S}\right\rangle=\left\langle u_{11}^{S}\right\rangle+\left\langle u_{22}^{S}\right\rangle+\left\langle u_{33}^{S}\right\rangle=0$ under static, laterally unconstrained conditions, the only independent strain is $u_{11}^{S}$. There is no dependence of $\left\langle\phi^{S}\right\rangle$ on $u_{22}^{S}$ and $u_{33}^{S}$. Thus, $\left\langle\sigma_{22}^{S}\right\rangle=\partial\left\langle\phi^{S}\right\rangle / \partial u_{22}^{S}=0$ and $\left\langle\sigma_{33}^{S}\right\rangle=$ $\partial\left\langle\phi^{S}\right\rangle / \partial u_{33}^{S}=0$, which are exactly the conditions that result in the null dilatation $\left\langle\Theta^{S}\right\rangle=$ 0 for isotropic materials with zero shear modulus, i.e., ideal fluids [66]. This means that the Lagrangian excess pressures normal to the plane wave propagation direction are obtained as

$$
\left\langle p_{2}^{L}\right\rangle-p_{0}=\left\langle p_{3}^{L}\right\rangle-p_{0}=-\left\langle\sigma_{22}^{S}\right\rangle+A_{1}^{S}=-\left\langle\sigma_{33}^{S}\right\rangle+A_{1}^{S}=0
$$

This also means, from Eq.(17) and the null value of the Lagrangian stresses in directions $\mathbf{e}_{2}$ and $\mathbf{e}_{3}$ under static conditions, that the Eulerian excess pressures normal to the wave 
propagation direction are given as

$$
\left\langle p_{2}^{E}\right\rangle-p_{0}=\left\langle p_{3}^{E}\right\rangle-p_{0}=0
$$

Thus, for laterally unconfined, plane wave propagation in fluids the acoustic radiation pressure along the direction of propagation is $\frac{1}{4}\langle 2 K\rangle$ and zero in the directions normal to the propagation direction. These results hold for both Lagrangian and Eulerian coordinates for laterally unconfined, plane wave propagation in fluids.

Eqs.(76) and (77) show that the mean Eulerian and Lagrangian excess pressures for laterally unconfined, plane waves are exactly equal with magnitude $(1 / 4)\langle 2 K\rangle$ along the direction of wave propagation. Again, these results are quite different from the Langevin expression for laterally unconfined, plane waves, which posits [27] that the mean Lagrangian pressure $\left\langle p_{1}^{L}\right\rangle$ along $\mathbf{e}_{\mathbf{1}}$ is obtained as

$$
\left\langle p_{1}^{L}\right\rangle=\langle K\rangle+\langle\phi\rangle+C=\langle 2 K\rangle+C=\langle E\rangle+C
$$

where it is assumed in Eq.(80) that $\langle 2 K\rangle=\langle E\rangle$ and $C=$ constant. It is appropriate to point out, in view of Eqs.(52) and (53), that the relation $\langle 2 K\rangle=\langle E\rangle$ is only approximately true for nonlinear waves. Nonetheless, in the interest of recounting the historical derivations, given in Section 4.2, the relation $\langle 2 K\rangle=\langle E\rangle$ is retained. Eq.(80) is known as Langevin's first relation. Assuming $C=p_{0}$ for plane wave propagation for laterally unconfined plane waves impinging on an absorptive target in a fluid medium leads to Langevin's result for the acoustic radiation pressure as

$$
\left\langle p_{1}^{\text {Langevin }}\right\rangle=\left\langle p_{1}^{L}\right\rangle-p_{0}=\langle E\rangle
$$

In view of the long-standing acceptance of the Langevin expression, Eq.(81), it is appropriate to analyze in some detail why the Langevin result differs from Eqs.(76) and (77). 


\subsection{Traditional derivations of radiation pressure for laterally, unconfined plane waves}

A number of efforts have been published in an attempt to validate analytically Langevin's result [27] that the acoustic radiation pressure in laterally unconfined, plane wave beams in fluids is equal to the mean total energy density, i.e. $\left\langle p_{1}^{\text {Langevin }}\right\rangle=\langle E\rangle$. The derivations do not distinguish analytically a priori, as done in Section 4.1, that the elastic properties associated with the radiation pressure result from laterally unconstrained, static conditions, while those of the driving acoustic wave result from laterally constrained, dynamic conditions. Recognition of the difference occurs a posteriori in the traditional derivations, which entail various erroneous and, in some cases, somewhat contrived arguments involving fluid flow to establish Langevin's result. Many efforts [26,32,38$40,46,47]$ begin by noting that for fluids the equation-of-state is expressed in terms of the mass density. The relation $\rho=\rho_{0}\left(1+u_{11}\right)^{-1}$ is expanded in terms of $u_{11}$, substituted in the equation-of-state for the fluid (a Eulerian equation), and time-averaged, assuming sinusoidal waves, to assess what is assumed to be the Lagrangian radiation pressure. The Eulerian radiation pressure is then assumed to be calculated by substituting the assessed Lagrangian pressure for the term $\left.q^{L}(X, t)\right|_{X=x}$ in the last equality in Eq.(3) and timeaveraging, again assuming sinusoidal waves. The difference between the calculations of the assumed Eulerian and Lagrangian radiation pressures is assessed to be the energy density, i.e. $\left\langle p_{1}^{L}\right\rangle-\left\langle p_{1}^{E}\right\rangle=\langle E\rangle=\langle 2 K\rangle$.

The relationship $\left\langle p_{1}^{L}\right\rangle-\left\langle p_{1}^{E}\right\rangle=\langle E\rangle=\langle 2 K\rangle$ is used to assess the Langevin radiation pressure defined $[46,47]$ "as the difference between the mean pressure at a reflecting or absorbing wall and the pressure in the un-perturbed fluid behind the wall, with the fluid being in contact with the two sides." This definition is equivalent to the condition that for laterally unconfined, plane waves the mean dilatation $\left\langle\Theta^{S}\right\rangle=0$, since "the fluid being in contact with the two sides" requires the lateral free flow of fluid. But the laterally unconfined, static condition is not analytically imposed a priori in the traditional derivations $[26,32,39,40]$. Instead, to obtain the Langevin result, $\left\langle p_{1}^{\text {Langevin }}\right\rangle=\langle E\rangle$, it is generally assumed that because the 'surfaces normal to the wave propagation direction $\mathbf{e}_{1}$ are oscillating,' the radiation pressure in that direction must correspond to the Lagrangian radiation pressure, and because the 'surfaces normal to the directions $\mathbf{e}_{2}$ and $\mathbf{e}_{3}$ are fixed,' the radiation pressure in the directions $\mathbf{2} 2$ and $\mathbf{e} 3$ must correspond to the Eulerian radiation 
pressure. Since the volume containing the wave is laterally unconfined, it is assumed in some approaches $[26,32,47]$ that the initial pressure and energy density inside the volume change to equalize the ambient pressure $p_{0}$ outside the volume. The equalization is used to obtain the Langevin pressure as $\left\langle p_{1}^{\text {Langevin }}\right\rangle=\left\langle p_{1}^{L}\right\rangle-\left\langle p_{1}^{E}\right\rangle=\langle 2 K\rangle=\langle E\rangle \quad$ (see, for example, $[26,32,47]$ for details).

There are two primary problems with the derivations outlined above. First, as pointed out in Section 2.4, it is not correct to assume that the Lagrangian coordinates are 'moving' coordinates and the Eulerian coordinates are 'fixed' coordinates, such that the coordinates necessary to describe motion along the direction of propagation must be Lagrangian coordinates and that the coordinates normal to the wave motion must be Eulerian coordinates. Either set of coordinates can be regarded as fixed or moving. One must decide in setting up and solving a given problem whether to work in Lagrangian or Eulerian coordinates, since it is improper to mix the coordinates arbitrarily. The second problem in the derivations is the employment of the power series expansion given in the last equality in Eq.(3) to assess the Eulerian radiation pressure from the Lagrangian pressure. The Eulerian pressure must be assessed from the transformation given by Eq.(12) or Eq.(17), which shows that for longitudinal, plane wave propagation the Lagrangian and Eulerian pressures are exactly equal, as given by Eq.(16). Moreover, the initial assumption that substituting the relation $\rho=\rho_{0}\left(1+u_{11}\right)^{-1}$ in an Eulerian equation-of-state leads to an expression for the Lagrangian pressure is itself incorrect. The substitution simply yields the Eulerian pressure written in terms of the displacement gradients [61].

Other attempts to assess the acoustic radiation pressure along the propagation direction for laterally unconfined, plane waves rely on establishing that the "mean excess Eulerian pressure,' $\left\langle p_{1}^{E}\right\rangle-p_{0}=-\left\langle T_{11}\right\rangle+\left(T_{11}\right)_{0}$, along the propagation direction $\mathbf{e}_{1}$ is zero, leaving the radiation pressure to be equal to the momentum flux density, $\left\langle\rho v_{1} v_{1}\right\rangle=$ $\langle E\rangle$, in the Brillouin stress tensor, Eq.(70). However, as shown in Section 3.2.2, the term identified in the Brillouin stress tensor as the momentum flux density is not the momentum flux density at all, but rather the contribution to the change in the system period resulting from the variation in the acoustic path length in response to the virtual variation in the strain, when the variation in the system period is assessed using the true velocity. And the term identified by Brillouin as the 'mean excess Eulerian pressure' is simply the 
contribution to the change in system period resulting from the change in the true sound velocity, when the variation in the system period is assessed using the true velocity.

Brillouin largely ignored the 'mean excess Eulerian pressure' term in applications of the Brillouin stress tensor, assuming the term to be irrelevant in assessing the radiation pressure. Several attempts to justify Brillouin's assumption have appeared in the literature. The approach to establishing a null 'mean excess Eulerian pressure' has been to utilize the relationship between pressure and enthalpy. The derivations of Lee and Wang [46]. Beissner [48], Beissner and Makarov [49], and Hasegawa et al. [45] are representative of such approaches. Consider the wave equation in Eulerian coordinates

$$
\rho\left(\frac{\partial u_{i}}{\partial t}+u_{j} \frac{\partial u_{i}}{\partial x_{j}}\right)=-\frac{\partial p^{E}}{\partial x_{i}}
$$

For irrotational sound waves $\boldsymbol{u}=\nabla \varphi^{S P}$, where $\varphi^{S P}$ is the scalar potential. Eq.(82) can thus be re-written as

$$
\nabla\left(\frac{\partial \varphi^{S P}}{\partial t}+\frac{1}{2}\left|\nabla \varphi^{S P}\right|^{2}\right)=-\frac{\nabla p^{E}}{\rho}
$$

It is generally assumed that Eq.(83) can be solved using the thermodynamic relationship $d H=\left(d p^{T h} / \rho_{0}\right)+T d S_{m}$ where $H=H\left(p^{T h}, S_{m}\right)$ is the enthalpy per unit mass, $S_{\mathrm{m}}$ is the entropy per unit mass, and $p^{T h}$ is the thermodynamic pressure $[55,58]$. It is critically important to recognize that the thermodynamic pressure $p^{T h}$ is not the Eulerian pressure $p^{E}$. The thermodynamic pressure is obtained from the thermodynamic tensions (second Piola-Kirchhoff stress) $t_{i j}=\rho_{0}\left(\partial U / \partial \eta_{i j}\right)$ via the relation $p^{T h} \delta_{i j}=-t_{i j}[55,58]$. For longitudinal, plane wave propagation along direction $\mathbf{e}_{1}, p_{1}^{L}=p_{1}^{E}=\left(1+u_{11}\right) p_{1}^{T h}$ $[55,58]$. It is generally overlooked that the pressure in the thermodynamic relationship for enthalpy is the thermodynamic pressure $p^{T h}$ and it is incorrectly assumed in Eq.(83) that for adiabatic motion $\nabla p^{E} / \rho=\nabla H$. It is then assumed that the pressure $p^{E}$ can be expanded in a power series in the enthalpy $H$ [46] or that $H-H_{0}=\int_{p_{0}}^{p^{E}} \rho^{-1} d p^{E}$ can be expanded in terms of the pressure $p^{E}[45,48,49]$. The power series expansion is a key operation in the derivations to obtain the relationship for propagation along e1 (see 
$[45,46,48,49]$ for details)

$$
\left\langle p_{1}^{E}\right\rangle=\langle\phi\rangle-\langle K\rangle+C
$$

where $C$ is a constant and $\langle\phi\rangle$ and $\langle K\rangle$ are, respectively, the time-averaged potential and kinetic energies of the wave.

Eq.(84) is known as Langevin's second relation and is generally regarded as the expression defining the mean Eulerian pressure in a wave. For plane, progressive waves it is traditionally assumed that $\langle\phi\rangle=\langle K\rangle$. If $\mathrm{C}$ is assumed to be the initial pressure $p_{0}$, Eq.(84) predicts that the mean excess Eulerian pressure is zero. This means, in regard to the traditional (incorrect) interpretation of the Brillouin stress tensor, that the acoustic radiation pressure depends only on the momentum flux density $\left\langle\rho v_{1} v_{1}\right\rangle=\langle E\rangle$ for laterally unconfined, plane wave propagation along $\mathbf{e}$.

The problem with the derivations leading to Eq.(84) is that the Eulerian pressure $p^{E}$ is not the thermodynamic pressure $p^{T h}$. Substituting $\nabla H$ for $\nabla p^{E} / \rho$ in Eq.(83) does not affect the terms on the left-hand side of the equation but it changes the right-hand side of the equation from a dependence on $p^{E}$ to a dependence on $p^{T h}$. By substituting $p^{T h}$ for $p^{E}$ in the relevant equations, the arguments of references $[45,46,48,49]$ lead to the relation

$$
\left\langle p_{1}^{T h}\right\rangle=\langle\phi\rangle-\langle K\rangle+C
$$

for propagation along direction e1, rather than to Eq.(84). For longitudinal wave propagation along $\mathbf{e}_{1}[55,58]$,

$$
\begin{gathered}
\left\langle p_{1}^{T h}\right\rangle=\left\langle\left(1+u_{11}\right)^{-1} p_{1}^{E}\right\rangle=\left\langle\left(1+u_{11}\right)^{-1} p_{1}^{L}\right\rangle \\
\approx\left\langle p_{1}^{E}\right\rangle-\left\langle u_{11} p_{1}^{E}\right\rangle=\left\langle p_{1}^{L}\right\rangle-\left\langle u_{11} p_{1}^{L}\right\rangle=\left\langle p_{1}^{E}\right\rangle+2\langle K\rangle .
\end{gathered}
$$

The last equality in Eq.(86) follows from the virial theorem, Eq.(50), where $\sigma_{11}=-p_{1}^{L}=$ $=-p_{1}^{E}$. Substituting Eq.(86) in Eq.(85) leads to

$$
\left\langle p_{1}^{E}\right\rangle=\left\langle p_{1}^{L}\right\rangle=\langle\phi\rangle-3\langle K\rangle+C
$$


Eq.(87) does not yield Langevin's second relation, Eq.(84).

The assumption that the enthalpy $H$ is a function of the Eulerian pressure $p^{E}$ has further implications regarding the expansion of $p^{E}$ in a power series in $H$. From Eq.(6), the internal energy per unit mass $U=U\left(\boldsymbol{X}, \eta_{i j}, S_{m}\right)$. For plane wave propagation along $\mathbf{e}_{\mathbf{1}}$

$$
p_{1}^{E}=p_{1}^{L}=\left(1+u_{11}\right) p_{1}^{T h}
$$

and

$$
d U=\left(\partial U / \partial \eta_{11}\right)_{S} \eta_{11}+(\partial U / \partial S)_{\eta} d S_{m}
$$

where $\left(\partial U / \partial \eta_{11}\right)_{S}=-p_{1}^{T h} / \rho_{0},\left(\partial U / \partial S_{m}\right)_{\eta}=T$, and $T$ is temperature [55]. The enthalpy per unit mass $H$ is related to the internal energy per unit mass $U$ as [55]

$$
H=H\left(p^{T h}, S_{m}\right)=U+\rho_{0}^{-1} \eta_{11} p^{T h}
$$

The differential of $H$ is

$$
d H=\left(\partial H / \partial p_{1}^{T h}\right)_{S} d p_{1}^{T h}+\left(\partial H / \partial S_{m}\right)_{p} d S_{m}
$$

where $\left(\partial H / \partial p^{T h}\right)_{S}=\rho_{0}^{-1} \eta_{11}$ and $\left(\partial H / \partial S_{m}\right)_{p} d S_{m}=T d S_{m}$. For present purposes, it is sufficient to consider only the linear approximation for isentropic conditions such that $p_{1}^{T h} \approx p_{1}^{L}=p_{1}^{E}, \eta_{11} \approx u_{11}$, and expand $p_{1}^{E}$ to first order in $H$ as

$$
\begin{gathered}
p_{1}^{T h}=p_{1}^{E}=p_{0}+\left(\frac{\partial p_{1}^{E}}{\partial H}\right)_{S_{m}, 0} H=p_{0}+\rho_{0} u_{11}^{-1}\left(U+\rho_{0}^{-1} u_{11} p_{1}^{E}\right) \\
=p_{0}+p_{1}^{E}+\rho_{0} u_{11}^{-1} U
\end{gathered}
$$

where the enthalpy-internal energy relationship [55] $H=U+\rho_{0}^{-1} u_{11} p_{1}^{T h}=U+$ $\rho_{0}^{-1} u_{11} p_{1}^{E}$ and the relation $\left(\partial p_{1}^{T h} / \partial H\right)_{S_{m}}=\left(\partial p_{1}^{E} / \partial H\right)_{S_{m}}=\rho_{0} u_{11}^{-1}$ in the linear 
approximations are substituted in Eq.(88) to obtain the last equality. Solving Eq.(88) for $U$ yields $U=-\left(p_{0} / \rho_{0}\right) u_{11}$. Since the pressure in the linear approximation is defined from the internal energy per unit mass as [55] $p_{1}^{E}=p_{1}^{T h}=-\rho_{0}\left(\partial U / \partial u_{11}\right)_{S_{m}}$, substituting $U=$ $-\left(p_{0} / \rho_{0}\right) u_{11}$ in the expression results in $p_{1}^{E}=p_{0}$. This states that the only term allowed in the expansion in Eq.(88) is the constant initial pressure $p_{0}$, which indicates that an expansion of the pressure in terms of the enthalpy is not a meaningful operation. This is not surprising, since, analogously, it is not generally meaningful to expand the strain in terms of the internal energy.

It is concluded that Langevin's second relation, Eq.(84), is incorrect and does not provide an assessment of the mean Eulerian pressure, as traditionally assumed. Indeed, the results of Eqs.(39), (40), (60), and (61) already suggest that since $\left\langle p_{1}^{E}\right\rangle=\left\langle p_{1}^{L}\right\rangle$ for plane wave propagation along e1, Langevin's second relation, $\left\langle p_{1}^{E}\right\rangle=\langle\phi\rangle-\langle K\rangle+C$, and Langevin's first relation, $\left\langle p_{1}^{L}\right\rangle=\langle\phi\rangle+\langle K\rangle+C$, cannot both be correct. Equally important, it is seen from Eqs.(76) and (77) that Langevin's first relation is also incorrect, as the equation does not account analytically a priori for the difference between the elastic properties under laterally unconfined, static conditions and that of the driving acoustic wave propagating under laterally confined, dynamic conditions.

\subsection{Implications for transducer calibration}

Issenmann et al. [50] point out that "despite the long-lasting theoretical controversies ... the Langevin radiation pressure ... has been the subject of very few experimental studies." Among the most important experimental studies are those by Herrey [51], who shows that the radiation pressure in laterally unconfined, plane wave beams in fluids is anisotropic, and Rooney [52], who shows that the radiation pressure in such beams is independent of the dynamic acoustic nonlinear parameter $\beta=$ $\left(-A_{111} / A_{11}\right)$ of the fluid - although Eqs.(72) show that the static nonlinearity parameter $\beta^{S}=\left(-A_{111}^{S} / A_{11}^{S}\right)$ for such beams in all fluids is one. Absolute measurements obtained independently in the same experiment for the power generated by an acoustic source and the radiation force incident on a target are generally lacking. Indeed, Beissner [21] points out that if acoustic radiation pressure is used to calibrate acoustic transducers the "measured radiation force must be converted to the ultrasonic power value and this is 
carried out with the help of theory." The measured radiation force on the target is generally assumed to result from the Langevin relation between the radiation pressure generated by the acoustic source and the energy density of the wave.

Consider a planar transducer of active area $\mathrm{S}_{\mathrm{A}}$ that emits an idealized plane wave. It is assumed for practical purposes that $\langle E\rangle=2\langle K\rangle$. The average ultrasonic power $\left\langle W_{p w r}\right\rangle$ emitted by the transducer is related to the energy density of the plane wave as $\left\langle W_{p w r}\right\rangle=$ $\langle E\rangle S_{A} c$, where $c$ is the sound velocity in the propagation medium. If Langevin's theory is assumed to be correct, then along the direction of plane wave propagation, the average force $\langle F\rangle$ generated over the area $S_{A}$ normal to the propagation direction is $\langle F\rangle=\langle E\rangle S_{A}$ and the average ultrasonic power is $\left\langle W_{p w r}^{\text {Langevin }}\right\rangle=\langle F\rangle_{c}$. In contrast, the present theory for laterally unconfined plane waves predicts from Eqs.(76) and (77) that $\left\langle p_{1}^{E}\right\rangle-p_{0}=$ $\left\langle p_{1}^{L}\right\rangle-p_{0}=\frac{1}{4}\langle 2 K\rangle=\frac{1}{4}\langle E\rangle$. The average force $\langle F\rangle$ generated by a plane wave propagating along $\mathbf{e}_{1}$ and normally incident on a surface of area $S_{A}$ is $\langle F\rangle=\left(\left\langle p_{1}^{E}\right\rangle-p_{0}\right) S_{A}=\left(\left\langle p_{1}^{L}\right\rangle-\right.$ $\left.p_{0}\right) S_{A}=\frac{1}{4}\langle E\rangle S_{A}$. Thus, $\langle E\rangle=4\langle F\rangle / S_{A}$ and the average ultrasonic power $\left\langle W_{p w r}\right\rangle$ generated by the transducer is assessed from the present theory to be

$$
\left\langle W_{p w r}\right\rangle=\langle E\rangle S_{A} c=4\langle F\rangle c=4\left\langle W_{p w r}^{\text {Langevin }}\right\rangle
$$

Eq.(89) states that the power emitted by the transducer is four times larger than the power $\left\langle W_{p w r}^{\text {Langevin }}\right\rangle=\langle F\rangle c$ assessed from the Langevin theory.

\section{Summary and conclusion}

The long-standing controversy regarding acoustic radiation pressure in laterally confined and unconfined, acoustic beams in fluids is re-examined from the perspective of finite deformation theory. Since most of the confusion and misunderstanding regarding acoustic radiation pressure stems from the seminal papers [23-28,38-40], which assume plane wave propagation in fluids, the present effort also focuses on plane wave propagation in fluids. One of the most important findings from finite deformation theory is that, for plane wave propagation, the Lagrangian and Eulerian pressures are exactly equal along the propagation direction. This is contrary to the result obtained in traditional derivations using 
an expansion of the Eulerian and Lagrangian pressures in terms of the particle displacement, given by Eqs.(3) and (4). It is shown in Section 2.4 that the expansions in Eqs.(3) and (4) do not define the transformation between Lagrangian and Eulerian quantities, as generally assumed, but simply provide an assessment of the difference between a given Lagrangian quantity, Eq.(3), or a given Eulerian quantity, Eq.(4), evaluated at two different points in Cartesian space, separated by the displacement $u$. The correct transformation between Lagrangian and Eulerian quantities must be obtained from the transformation coefficients of finite deformation theory, as discussed in Section 2 and illustrated in Appendices A and B.

The traditional transformation between Lagrangian and Eulerian quantities obtained from the expansions in Eqs.(3) and (4) has been motivated in part by the incorrect assumptions that (a) surfaces normal to the direction $\mathbf{e}_{1}$ of wave propagation oscillate and, therefore, the radiation pressure along that direction must be the Lagrangian radiation pressure, and (b) surfaces normal to the directions $\mathbf{e}_{2}$ and $\mathbf{e}_{3}$ are fixed and, therefore, the radiation pressure in those directions must be the Eulerian radiation pressure. As shown in Section 2.4, either set of coordinates may be regarded as fixed. For work in solids it the Lagrangian coordinates that are usually regarded as fixed [42,43,55-57,60]. It is improper and somewhat contrived to mix coordinate systems arbitrarily in a given problem, especially to assign a particular coordinate system to physical motion based on the assumed attributes of the coordinate system regarding that motion. Such assigning of attributes and mixing of Lagrangian and Eulerian coordinates have led to considerable misunderstanding and confusion in assessing the acoustic radiation pressure.

A laterally confined, plane wave propagating along direction $\mathbf{e}_{1}$ corresponds to the condition that the lateral strains (displacement gradients) $u_{22}=u_{33}=0$. Such a condition applies to a plane wave of infinite cross-section or to an idealized plane wave propagating in a laterally enclosed volume with infinitely stiff lateral boundaries. The application of finite deformation theory leads to the traditionally derived Rayleigh radiation pressure $\left\langle p_{1}^{\text {Rayleigh }}\right\rangle=\frac{1}{4}\left(\frac{B}{A}+2\right)\langle 2 K\rangle$ for liquids and $\left\langle p_{1}^{\text {Rayleigh }}\right\rangle=\frac{1}{4}(\gamma+1)\langle 2 K\rangle$ for ideal gases along the propagation direction $\mathbf{e}_{\mathbf{1}}$ for both Lagrangian and Eulerian coordinates. In directions $\mathbf{e}_{2}$ and $\mathbf{e}_{3}$ the radiation pressure is given in Lagrangian coordinates as $\left\langle p_{2}^{L}\right\rangle-$ 
$p_{0}=\left\langle p_{3}^{L}\right\rangle-p_{0}=\frac{1}{4}\left(\frac{B}{A}-2\right)\langle 2 K\rangle$ for liquids, assuming $p_{0} / A \ll<1$. The identical results were also derived by Brillouin from the Boltzmann-Ehrenfest Principle of Adiabatic Invariance (after correcting for an omitted nonlinear term in Brillouin's derivation), but Brillouin posited that the results are in Eulerian coordinates rather than, as shown in Section 3.2.2, in Lagrangian coordinates.

It is shown in Section 3.2.2 that Brillouin misidentified two terms in the equation for the radiation pressure obtained from the Boltzmann-Ehrenfest Adiabatic Principle. One term, which Brillouin called the momentum flux density, is not the momentum flux density at all but the contribution to the fractional variation in the period of wave oscillation resulting from a change in the path length from slow, virtual variations in the strain (generalized displacement in terms of the Boltzmann-Ehrenfest Principle). The second term misidentified by Brillouin is the contribution to the fractional change in the system period associated with the change in the true sound velocity from virtual variations in the strain. Brillouin called this latter term the 'mean Eulerian excess pressure,' $\left\langle p_{1}^{E}\right\rangle-p_{0}$, and called the sum of the two misidentified terms the components of an entirely new stress tensor, known today as the Brillouin stress tensor. It is shown in Section 3.2.2 that the components of the Brillouin stress tensor are actually the components of the Lagrangian radiation stress tensor for laterally confined, plane waves propagating in direction $\mathbf{e}$.

A laterally unconfined, plane wave propagating along direction $\mathbf{e}_{1}$ in fluids corresponds to the condition that the mean stresses along the directions $\mathbf{e}_{2}$ and $\mathbf{e}_{3}$ are zero or, equivalently, that the mean dilatation $\left\langle\Theta^{S}\right\rangle$ under the static (time-averaged, steady-state) conditions associated with the radiation pressure is given by $\left\langle\Theta^{S}\right\rangle=\left\langle\Delta V^{S} / V_{0}^{S}\right\rangle=\left\langle u_{11}^{S}\right\rangle+$ $\left\langle u_{22}^{S}\right\rangle+\left\langle u_{33}^{S}\right\rangle=0$, where $\left\langle\Delta V^{S}\right\rangle$ is the mean change in the initial volume $V_{0}^{S}$ [66]. The null mean dilatation results from the null shear modulus that is characteristic of fluids [66]. The null mean dilatation and the symmetry along $\mathbf{e}_{2}$ and $\mathbf{e} 3$ reduce the number of independent strain variables to one, chosen under static conditions to be $u_{11}^{S}$. It is shown in Section 4.1 from finite deformation theory and from the Boltzmann-Ehrenfest Adiabatic Principle that for both Eulerian and Lagrangian coordinates the acoustic radiation pressure for plane waves along the direction of propagation is given as $\left\langle p_{1}^{E}\right\rangle-p_{0}=\left\langle p_{1}^{L}\right\rangle-p_{0}=$ $\frac{1}{4}\langle 2 K\rangle \approx \frac{1}{4}\langle E\rangle$. Normal to the wave propagation direction $\left\langle p_{2}^{E}\right\rangle-p_{0}=\left\langle p_{2}^{L}\right\rangle-p_{0}=$ 
$\left\langle p_{3}^{E}\right\rangle-p_{0}=\left\langle p_{3}^{L}\right\rangle-p_{0}=0$. The results are the direct consequence of the a priori assumptions in the derivation of a null mean dilatation, the directional symmetry for plane wave propagation, and the application of the relationship between the Huang elastic coefficients and the Fox-Wallace-Beyer coefficients for liquids (ratio of specific heats for gases) obtained from Eqs.(26)-(31).

The present results for laterally unconfined plane wave propagation in fluids are contrary to the traditional results obtained both from the Brillouin theory, where the radiation pressure is assumed to result from the momentum flux density $\left\langle\rho v_{1} v_{1}\right\rangle$, and the Langevin theory, which predicts that $\left\langle p_{1}^{\text {Langevin }}\right\rangle=\langle E\rangle$ along the wave propagation direction. As examined analytically in Section 4.2, the traditional derivations for laterally unconfined, plane waves in fluids do not account a priori for the difference between the elastic properties under static, laterally unconfined conditions (giving rise to free fluid flow) and that of the driving acoustic wave propagating under laterally confined conditions (that do not permit free flow). Rather than accounting a priori for the difference in elastic properties, a patchwork of a posteriori assumptions, definitions, and arguments has been used in various attempts to quantify the radiation pressure for laterally unconfined, plane waves. The derivations are typically based on a number of misconceptions that have permeated the acoustics literature including (a) a widespread misunderstanding of Lagrangian and Eulerian quantities and of the transformation between them, (b) the misinterpretation by Brillouin of terms leading to the Brillouin stress tensor, and (c) the assumption that the pressure defined by the enthalpy in deriving Langevin's second relation is the Eulerian pressure rather than the thermodynamic pressure (second Piola-Kirchhoff pressure). The present work corrects these misconceptions and provides a coherent, first principles examination of acoustic radiation pressure based on finite deformation theory that is independently verified from the Boltzmann-Ehrenfest Principle of Adiabatic Invariance.

The acoustic radiation pressure is used in a variety of applications [1-22], many of which rely on a reliable assessment of the force on a target generated by an acoustic source. In such cases, as pointed out by Beissner [21], the "measured radiation force must be converted to the ultrasonic power value and this is carried out with the help of theory." It is appreciated that the measurements for diffracted and focused beams are not described 
by simple plane wave propagation, but because of the large difference between the present value of $(1 / 4)\langle 2 K\rangle$ for the acoustic radiation pressure along the propagation direction for laterally unconfined, plane waves and the value $\langle E\rangle \approx\langle 2 K\rangle$ from the Langevin theory or $\left\langle\rho v_{1} v_{1}\right\rangle$ from the Brillouin stress tensor, it would seem prudent to re-examine relevant applications in view of the present theoretical results. The use of the Langevin theory is of particular concern when acoustic radiation pressure is used to calibrate transducers. Indeed, the power $\left\langle W_{p w r}\right\rangle$ emitted from the transducer as assessed from the present theory is $\left\langle W_{p w r}\right\rangle=4\langle F\rangle c$, where $\langle F\rangle=$ the average force and $c=$ sound velocity. This value is four times larger than the power $\left\langle W_{p w r}^{\text {Langevin }}\right\rangle=\langle F\rangle c$ predicted by the Langevin theory.

\section{Acknowledgement}

The author thanks Dr. S. A. Cantrell for useful discussions.

\section{Appendix A. Mass density in Lagrangian and Eulerian coordinates}

Consider two points in the Lagrangian (rest or initial) coordinates in a material given by the vectors $\boldsymbol{X}^{(\mathrm{A})}$ and $\boldsymbol{X}^{(\mathrm{B})}$ separated by the infinitesimal vector distance $\mathrm{d} \boldsymbol{X}=\boldsymbol{X}^{(\mathrm{B})}$ $-\boldsymbol{X}^{(\mathrm{A})}$. During deformation, points $\boldsymbol{X}^{(\mathrm{A})}$ and $\boldsymbol{X}^{(\mathrm{B})}$, respectively, move to points $\boldsymbol{x}^{(\mathrm{A})}$ and $\boldsymbol{x}^{(\mathrm{B})}$ in the Eulerian (present) coordinates, separated by the vector displacement $\mathrm{d} \boldsymbol{x}=\boldsymbol{x}^{(\mathrm{B})}-\boldsymbol{x}^{(\mathrm{A})}$. The deformation $d \boldsymbol{X} \rightarrow d \boldsymbol{x}$ is given as

$$
d x_{i}=x_{i}\left(\boldsymbol{X}^{(\boldsymbol{A})}+d \boldsymbol{X}\right)-x_{i}\left(\boldsymbol{X}^{\boldsymbol{A}}\right)=\alpha_{i j} d X_{j}
$$

where $\alpha_{i j}=\partial x_{i} / \partial X_{j}$ are the transformation coefficients of finite deformation theory. Eq.(A1) can be used to obtain the relationship between the un-deformed mass density $\rho_{0}$ and the deformed mass density $\rho$ by assuming that the specific volume containing the mass in the un-deformed state is a rectangular parallelepiped with sides parallel to the Cartesian coordinate axes having unit vectors $\mathbf{e}_{1}, \mathbf{e}_{2}$, and $\mathbf{e}_{3}$. Thus, $\mathrm{d} \boldsymbol{X}^{(1)}=\left|\mathrm{d} \boldsymbol{X}^{(1)}\right| \mathbf{e}_{1}, \mathrm{~d} \boldsymbol{X}^{(2)}=\left|\mathrm{d} \boldsymbol{X}^{(2)}\right| \mathbf{e}_{2}$, $\mathrm{d} \boldsymbol{X}^{(3)}=\left|\mathrm{d} \boldsymbol{X}^{(3)}\right| \mathbf{e}_{3}$ and the elemental un-deformed specific volume $\mathrm{d} V_{0}$ in the Lagrangian coordinates is obtained as 


$$
\begin{gathered}
d V_{0}=d \boldsymbol{X}^{(1)} \cdot d \boldsymbol{X}^{(2)} \times d \boldsymbol{X}^{(3)}=\left|d X^{(1)}\right|\left|d X^{(2)}\right|\left|d X^{(3)}\right| \boldsymbol{e}_{1} \cdot \boldsymbol{e}_{2} \times \boldsymbol{e}_{3} \\
=\left|d X^{(1)}\right|\left|d X^{(2)} \| d X^{(3)}\right|
\end{gathered}
$$

From Eq.(A1) the Lagrangian vector $\mathrm{d} \boldsymbol{X}^{(1)}$ deforms to a vector $\mathrm{d} \boldsymbol{x}^{(1)}=\left|\mathrm{d} \boldsymbol{x}^{(1)}\right| \mathbf{m}_{1}\left(\mathbf{m}_{1}\right.$ $=$ unit vector $)$ in Eulerian coordinates with Cartesian components $\mathrm{d}\left(\boldsymbol{x}^{(1)}\right)_{\mathrm{i}}=\alpha_{\mathrm{i} 1}\left|\mathrm{~d} \boldsymbol{X}^{(1)}\right|(\mathrm{i}=1$, 2, 3), $\mathrm{d} \boldsymbol{X}^{(2)}$ deforms to $\mathrm{d} \boldsymbol{x}^{(2)}=\left|\mathrm{d} \boldsymbol{x}^{(2)}\right| \mathbf{m}_{2}\left(\mathbf{m}_{2}=\right.$ unit vector) with components $\mathrm{d}\left(\boldsymbol{x}^{(2)}\right)_{\mathrm{i}}=$ $\alpha_{\mathrm{i} 2}\left|\mathrm{~d} \boldsymbol{X}^{(2)}\right|(\mathrm{i}=1,2,3)$, and $\mathrm{d} \boldsymbol{X}^{(3)}$ deforms to $\mathrm{d} \boldsymbol{x}^{(3)}=\left|\mathrm{d} \boldsymbol{x}^{(3)}\right| \mathbf{m}_{3}\left(\mathbf{m}_{3}=\right.$ unit vector) with components $\mathrm{d}\left(\boldsymbol{x}^{(3)}\right)_{\mathrm{i}}=\alpha_{\mathrm{i} 3}\left|\mathrm{~d} \boldsymbol{X}^{(3)}\right|(\mathrm{i}=1,2,3)$. The elemental deformed specific volume $d V=$ $d \boldsymbol{x}^{(1)} \cdot d \boldsymbol{x}^{(2)} \times d \boldsymbol{x}^{(3)}$ is thus related to the elemental un-deformed specific volume $\mathrm{d} V_{0}$ as

$$
\frac{d V}{d V_{0}}=\frac{d \boldsymbol{x}^{(1)} \cdot d \boldsymbol{x}^{(2)} \times d \boldsymbol{x}^{(3)}}{d \boldsymbol{X}^{(1)} \cdot d \boldsymbol{X}^{(2)} \times d \boldsymbol{X}^{(13)}}=\operatorname{det} \alpha_{i j} \equiv J=\frac{\rho_{0}}{\rho}
$$

where $J$ is the Jacobian determinant, $\operatorname{det}\left(\alpha_{\mathrm{ij}}\right)$, of the transformation. The last equality in Eq.(A3) follows from the fact that the mass contained in the un-deformed and deformed specific volumes remains constant during deformation.

Although the mass density $\rho$ in Eq.(A3) is expressed in terms of the transformation coefficients $\alpha_{i j}$, which refer to Lagrangian coordinates, it is important to recognize that at a given point and time in Cartesian space the Lagrangian and Eulerian mass densities, $\rho^{L}$ and $\rho^{E}$, respectively, are equal such that $\rho=\rho^{L}=\rho^{E}$. See Sections 2.2 and 2.3 for details.

\section{Appendix B. Lagrangian and Eulerian stresses and pressures}

Consider in the initial (rest) state of the material a plane of infinitesimal area $\mathrm{dS}_{0}$ with unit normal vector $\mathbf{N}$. The plane can be described in terms of the Lagrangian (initial) coordinates by the relation

$$
N_{i} d X_{i}=0
$$

where $i=1,2,3$ and $N_{\mathrm{i}}$ are the Cartesian components of $\mathbf{N}$. Under an impressed stress the planar area $\mathrm{dS}_{0}$ will be deformed into the infinitesimal planar area $\mathrm{d} S$ with unit normal vector $\mathbf{n}$, which by substituting the relation $d X_{i}=\gamma_{i j} d x_{j}$ in Eq.(B1) can be described in terms of the Eulerian (present) coordinates as $n_{\mathrm{j}} \mathrm{d} x_{\mathrm{j}}=0$ where the Cartesian components of 
n are

$$
n_{j}=\frac{N_{i} \gamma_{i j}}{f_{N}}
$$

The factor $f_{N}$ in Eq.(B2) is inserted to normalize $\mathbf{n}$. Normalization requires that $\mathrm{n}_{\mathrm{j}} \mathrm{n}_{\mathrm{j}}=1$, which yields the relation

$$
f_{N}^{2}=\gamma_{i k} \gamma_{j k} N_{i} N_{j} \approx\left(\delta_{i j}-2 \frac{\partial u_{i}}{\partial X_{j}}\right) N_{i} N_{j}
$$

From Eq.(A3) let $d \boldsymbol{x}^{(2)} \times d \boldsymbol{x}^{(3)}=d S \boldsymbol{n}$ where $d S$ is an infinitesimal area with unit normal vector $\mathbf{n}$ that results from the deformation of the infinitesimal area $d S_{0}=$ $\left|\mathrm{d} \boldsymbol{X}^{(2)} \| \mathrm{d} \boldsymbol{X}^{(3)}\right|$ in the $X_{2}-X_{3}$ plane with unit normal vector $\mathbf{e}_{1}$. Writing $\mathrm{d} \boldsymbol{x}^{(1)}=\left|\mathrm{d} \boldsymbol{x}^{(1)}\right| \mathbf{m}_{1}$, where $\mathbf{m}_{1}$ is a unit vector, yields the Eulerian specific volume element $d V$ as

$$
d V=d \boldsymbol{x}^{(1)} \cdot d \boldsymbol{x}^{(2)} \times d \boldsymbol{x}^{(3)}=\left|d \boldsymbol{x}^{(1)}\right| \boldsymbol{m}_{1} \cdot \boldsymbol{n} d S=d L d S,
$$

where $d L$ is the projection of $\mathrm{d} \boldsymbol{x}^{(1)}=\left|\mathrm{d} \boldsymbol{x}^{(1)}\right| \mathbf{m}_{1}$ along the direction $\mathbf{n}$ obtained from Eqs.(A1) and (B4) as

$$
d L=d \boldsymbol{x}^{(1)} \cdot \boldsymbol{n}=\left|d \boldsymbol{X}^{(1)}\right| \alpha_{j 1} \gamma_{i j} N_{i} / f_{N}=\left|d \boldsymbol{X}^{(1)}\right| / f_{N} .
$$

Writing $\ell=d L$ and $\ell_{0}=\left|d X^{(1)}\right|$ in Eq.(B5) and using the definition of $f_{N}$ given in Eq.(B3) lead to the relation

$$
R=\frac{\ell_{0}}{\ell}=f_{N}=\left[\left(\delta_{i j}-2 \frac{\partial u_{i}}{\partial X_{j}}\right) N_{i} N_{j}\right]^{1 / 2} .
$$

From Eq.(A3) and Eq.(B5) for $d L$ the ratio of the Eulerian specific volume element $d V$ to the Lagrangian specific volume element $d V_{0}$ is obtained as

$$
d V / d V_{0}=d L d S /\left(\left|d \boldsymbol{X}^{(1)}\right| d S_{0}\right)=d S /\left(d S_{0} f_{N}\right)=J,
$$

which leads to

$$
d S / d S_{0}=J f_{N} .
$$


A stress is defined as a force per unit area. It is noted that while the strain is defined with respect to the initial state of the material (i.e., with respect to the Lagrangian coordinates), the force $F_{\mathrm{i}}$ is usually defined with respect to a unit area of deformed material (i.e., with respect to the Eulerian coordinates) [55]. An exception is the second PiolaKirchhoff stress for which the force is referred to the undeformed state (Lagrangian coordinates) [55]. For acoustic wave propagation, the Cauchy (Eulerian) stress and first Piola-Kirchhoff (Lagrangian) stress are generally used. The force per unit area referred to the present configuration is called the Eulerian or Cauchy stress $T_{\mathrm{ij}}$. It is a force per unit area for which both the force and the area are referred to the deformed state $\boldsymbol{x}$ [55-60]. The Cauchy stress is symmetric and the i-component of the force $F_{\mathrm{i}}$ in Eulerian coordinates is $F_{\mathrm{i}}=T_{\mathrm{ji}} n_{\mathrm{j}} d S=T_{\mathrm{ij}} n_{\mathrm{j}} d S$ where $\mathbf{n}$ is the unit vector normal to the surface area $d S$. The force per unit area referred to the initial configuration is called the Lagrangian or first PiolaKirchhoff stress $\sigma_{\mathrm{ij}}$. It is a stress for which the force is referred to the deformed state $\boldsymbol{x}$ but the area is referred to the initial state $\boldsymbol{X}$ of the material [55-60]. The i-component of force in Lagrangian coordinates is obtained as $\sigma_{\mathrm{ik}} N_{\mathrm{k}} d S_{0}$ where $\mathbf{N}$ is the unit vector normal to the surface area $d S_{0}$. Since the force $F_{\mathrm{i}}$ is defined with respect to a unit area of deformed material (i.e., with respect to the Eulerian coordinates), this imposes that the relationship between the Lagrangian and Eulerian stresses is governed by the relationship between the surface areas $d S$ and $d S_{0}$.

Since the i-component of the force in Lagrangian coordinates is $\sigma_{\mathrm{ik}} N_{\mathrm{k}} d S_{0}$ and the force $F_{\mathrm{i}}=T_{\mathrm{ji}} n_{\mathrm{j}} d S$ is defined with respect to the Eulerian coordinates, it is appropriate to write the i-component of the force per area $d S_{0}$ in Lagrangian coordinates as

$$
\sigma_{i k} N_{k}=\frac{F_{i}}{d S_{0}}=T_{j i} n_{j} \frac{d S}{d S_{0}}=J f_{N} T_{j i} n_{j}=J \gamma_{k j} T_{j i} N_{k}
$$

where Eqs.(B2) and (B8) have been used to obtain the last two equalities in Eq.(B9). From the first and last equalities in Eq.(B9) the relationship between the Lagrangian stress $\sigma_{\mathrm{ik}}$ and Eulerian stress $T_{\mathrm{ij}}=T_{\mathrm{ji}}$ is obtained as 


$$
\sigma_{i k}=J \gamma_{k j} T_{j i}
$$

or equivalently

$$
T_{i j}=\frac{1}{J} \alpha_{i k} \sigma_{j k} .
$$

Eqs.(B10) and (B11) are in agreement with previous derivations using other (mathematically equivalent) approaches [55-60]. The equations are re-derived here to emphasize the importance of using the transformation coefficients $\alpha_{\mathrm{ij}}$ and $\gamma_{\mathrm{ij}}$ in establishing the connection between the Lagrangian and Eulerian stresses and pressures. Eqs.(B10) and (B11) also hold for the dynamical case of acoustic wave propagation, since for non-uniform deformations the transformation is considered to be local in $\boldsymbol{X}$ at time $t$ such that $\boldsymbol{x}=\boldsymbol{x}(\boldsymbol{X}$, $t$ ). Thus, the derivation also clearly shows that, contrary to a commonly held assumption, the Cauchy stress is the stress referred to Eulerian coordinates and when time-averaged is the acoustic radiation stress in Eulerian coordinates. Finally, it is assumed that the coordinate transformation is between the initial state and the final (present) state of the material and that the initial state may result from a constant applied or residual stress, or initial pressure.

\section{References}

1. M. L. Palmeri and K. R. Nightingale, Acoustic radiation force-based elasticity imaging methods, Interface Focus 1, 553-564 (2011).

2. J. R. Doherty, G. E. Trahey, K. R. Nightingale, and M. L. Palmeri, Acoustic radiation force elasticity imaging in diagnostic ultrasound, IEEE Trans. Ultrason. Ferroelectr. Freq. Control 60, 685-701 (2013).

3. F. Piscaglia, V. Salvatore, R. Di Donato, M. D’Onofrio, S. Gualandi, A. Gallotti, E. Peri, A. Borghi, F. Conti, G. Fattovich, E. Sagrini, A. Cucchetti, P. Andreone, and L. Bolondi, Accuracy of VirtualTouch Acoustic Radiation Force Impulse (ARFI) imaging for the diagnosis of cirrhosis during liver ultrasonography, Ultraschall. Med. 32,167175 (2011).

4. C. Amador, M. W. Urban, S. Chen, and J. F. Greenleaf, Loss tangent and complex modulus estimated by acoustic radiation force creep and shear wave dispersion, Phys. Med. Biol. 57, 1263-1282 (2012). 
5. J.-L Gennisson, T. Deffieux, M. Fink, and M. Tanter, Ultrasound elastography: principles and techniques, Diagnostic and Interventional Imaging 94, 487- 495 (2013).

6. M. Couade, M. Pernot, C. Prada, E. Messas, J. Emmerich, P. Bruneval, A. Criton, M. Fink, and M. Tanter, Quantitative assessment of arterial wall biomechanical properties using shear wave imaging, Ultrasound in Medicine and Biology 10, 1662-1676 (2010).

7. E. Bossy, A. R. Funke, K. Daoudi, A.-C. Boccara, M. Tanter, and M. Fink, Transient optoelastography in optically diffusive media, Appl. Phys. Lett. 90, 174111 (2007).

8. S. R. Aglyamov, A. B. Karpiouk, Y. A. Ilinskii, E. A. Zabolotsakya, and S. Y. Emelianov, Motion of a solid sphere in a viscoelastic medium in response to applied acoustic radiation force: Theoretical analysis and experimental verification, J. Acoust. Soc. Am. 122, 1927-1936 (2007).

9. A. B. Karpiouk, S. R. Aglyamov, Y. A. Ilinskii, E. A. Zaboloskaya, and S. Y. Emelianov, Assessment of shear modulus of tissue using ultrasound radiation force acting on a spherical acoustic inhomogeneity, IEEE Trans. Ultrason. Ferroelectr. Freq. Control 56, 2380-2387 (2009).

10. M. J. Shortencarrier, P. A. Dayton, S. H. Bloch, P. A. Schumann, T. O. Matsunaga, and K. W. Ferrara, A method for radiation-force localized drug delivery using gasfilled lipospheres, IEEE Trans. Ultrason. Ferroelectr. Freq. Control 57, 822-831 (2004).

11. P. L. Marston and D. B. Theissen, Manipulation of fluid objects with acoustic radiation pressure, Annals of the New York Academy of Sciences 1027, 414-434 (2004).

12. L. Zhang and P. L. Marston, Optical theorem for acoustic non-diffracting beams and application to radiation force and torque, Biomed. Opt. Express. 4, 1610-1617 (2013).

13. D. Baresch, J.-L. Thomas, and R. Marchiano, Observation of a single-beam gradient force acoustical trap for elastic particles: acoustical tweezers, Phys. Rev. Lett. 116, 024301 (2016).

14. C. E. M. Démoré, P. M. Dahl, Z. Yang, P. Glynne-Jones, A. Melzer, S. Cochran, M. P. MacDonald, and G. C. Spalding, Acoustic tractor beam, Phys. Rev. Lett. 112, 174302 (2014).

15. C. Spalding, Mechanical evidence of the orbital angular momentum to energy ratio of 
vortex beams, Phys. Rev. Lett. 108, 194301 (2012).

16. F. L. Degertekin, B. Hadimioglu, T. Sulchek, and C. F. Quate, Actuation and characterization of atomic force microscope cantilevers in fluids by acoustic radiation pressure, Appl. Phys, Lett. 78,1628-1630 (2001).

17. A. G. Onaran and F. L. Degertekin, A fluid cell with integrated acoustic radiation pressure actuator for atomic force microscopy, Rev. Sci. Instrum. 76, 103703 (2005).

18. M. Greenspan, F. R. Breckenridge, and C. E. Tschiegg, Ultrasonic transducer power output by modulated radiation pressure, J. Acoust. Soc. Am. 63, 1031-1038 (1978).

19. S. E. Fick, Ultrasound power measurement by pulsed radiation pressure, Metrologia 36, 351-356 (1999).

20. K. Beissner, Report on key comparison CCAUV.U-K1 (ultrasonic power), Metrologia 39, Tech. Suppl., 09001 (2002).

21. K. Beissner, Primary measurement of ultrasonic power and dissemination of ultrasonic power reference values by means of standard transducers, Metrologia 36, 313-320 (1999).

22. K. M. Swamy, F. J. Keil, Ultrasonic power measurements in the milliwatt region by the radiation force float method, Ultrasonics Sonochemistry 9, 305-310 (2002).

23. J. W. Strutt (Lord Rayleigh), On the momentum and pressure of gaseous vibrations and on the connexion with the virial theorem, Phil. Mag. 10, 364-74 (1905).

24. L. Brillouin, Tensors in Mechanics and Elasticity (Academic, New York, 1964), pp. 1- 478. (Translation of Les tenseurs en mécanique et en élasticité (Masson, Paris, 1938)).

25. L. Brillouin, Sur les tensions de radiation, Ann. Phys. 4, 528-586 (1925).

26. G. Hertz and H. Mende, Der Schallstrahlungsdruck in Flüssigkeiten, Z. Physik 114, 354-367 (1939).

27. P. A. Langevin (P. Biquard), Les ondes ultra-sonores, Rev. d'Acoust. 1, 93-109 (1932).

28. P. A. Langevin (P. Biquard), Les ondes ultra-sonores. II, Rev. d'Acoust. 1, 315-335 (1932).

29. F. E. Borgnis, Acoustic radiation pressure of plane compressional waves, Rev. Mod. Phys. 25, 653-664 (1953). 
30. Z. A. Goldberg, Acoustic radiation pressure: in High-Intensity Ultrasonic Fields, edited by L. D. Rozenburg (Plenum, New York, 1971), pp 75-133.

31. J.A. Rooney and W.L. Nyborg, Acoustic radiation pressure in a travelling plane wave, Am. J. Phys. 40, 1825-1830 (1972).

32. R.T. Beyer, Nonlinear Acoustics (Acoustical Society of America, Woodbury,1960), pp. 221-268.

33. B.-T. Chu and R. E. Apfel, Acoustic radiation pressure produced by a beam of sound, J. Acoust. Soc. Am. 72, 1673-87 (1982).

34. W. L. Nyborg, Comment on a paper by Chu and Apfel, J. Acoust. Soc. Am. 75, 263264 (1984).

35. B.-T. Chu, Relationship between Boussinesq and Cauchy radiation stresses in solids, Phys. Rev. B 25, 870-871 (1987).

36. G. T. Silva, An expression for the radiation force exerted by an acoustic beam with arbitrary wavefront, J. Acoust. Soc. Am. 130, 3541-3544 (2011).

37. G. T. Silva, Acoustic radiation force and torque on an absorbing compressible particle in an inviscid fluid, J. Acoust. Soc. Am. 136, 2405-2413 (2014).

38. F. V. Hunt, Notes on the exact equations governing the propagation of sound in fluids, J. Acoust. Soc. Am. 27, 1019 -1039 (1955).

39. P. J. Westervelt, The mean pressure and velocity in a plane acoustic wave in a gas, J. Acoust. Soc. Am. 22, 319-327 (1950).

40. P. J. Westervelt, The theory of steady forces caused by a sound wave, J. Acoust. Soc. Am. 23, 312-315 (1951).

41. D. T. Blackstock, Lagrangian one-dimensional equations of hydrodynamics for a viscous, thermally conducting fluid, J. Acoust. Soc. Am. 33, 1245-1246 (1961).

42. J. H. Cantrell, Acoustic radiation stress in solids I. theory, Phys. Rev. B 30, 3214-3220 (1984).

43. W.T. Yost and J.H. Cantrell, Acoustic-radiation stress in solids II. experiment, Phys. Rev. B 30, 3221-3227 (1984).

44. K. Beissner, The acoustic radiation force in lossless fluids in Eulerian and Lagrangian coordinates, J. Acoust. Soc. Am. 103, 2321-2332 (1998).

45. T. Hasegawa, T. Kido, T. Iizuka, and C. Matsuoka, A general theory of Rayleigh and 
Langevin radiation pressures, J. Acoust. Soc. Jpn. 21, 145-152 (2000).

46. C. P. Lee and T. G. Wang, Acoustic radiation pressure, J. Acoust. Soc. Am. 94, 10991109 (1993).

47. R. T. Beyer, Radiation pressure - the theory of a mislabeled tensor, J. Acoust. Soc. Am. 63, 2035-30 (1978).

48. K. Beissner, Two concepts of acoustic radiation pressure, J. Acoust. Soc. Am. 79, 16101612 (1978).

49. K. Beissner and S. N. Makarov, Acoustic energy quantities and radiation force in higher approximation, J. Acoust. Soc. Am. 97, 898-905 (1995).

50. B. Issenmann, A. Nicolas, R. Wunenburger, S. Manneville, J.-P. Delville, Deformation of acoustically transparent fluid interfaces by the acoustic radiation pressure, EPL Europhysics Letters 83, 34002 (2008).

51. E. M. J. Herrey, Experimental studies on acoustic radiation pressure, J. Acoust. Soc. Amer. 27, 891-896 (1955).

52. J. A. Rooney, Does radiation pressure depend on B/A, J. Acoust. Soc. Am. 54, 429430 (1973).

53. F.D. Murnaghan, Finite Deformation of an Elastic Solid (Wiley, New York, 1951), pp. 1 - 140.

54. C. Truesdell, General and exact theory of waves in finite elastic strain, Arch. Rational Mech. Anal. 8, 263-296 (1961).

55. R. N. Thurston, Wave propagation in fluids and normal solids: in Physical Acoustics, vol. I, edited by W.P. Mason, (Academic, New York, 1964), pp. 1-110.

56. R.N. Thurston and K. Brugger, Third-order elastic constants and the velocity of small amplitude elastic waves in homogeneously stressed media, Phys. Rev. 133, A1604A1610 (1964).

57. R.N. Thurston and M.J. Shapiro, Interpretation of ultrasonic experiments on finite amplitude waves, J. Acoust. Soc. Am. 41, 1112-1125 (1967).

58. C. Truesdell and R. A. Toupin, The classical field theories: in Handbuch der Physik, Vol. III/1, edited by S. Flugge, (Springer-Verlag, Berlin, 1960), pp. 236-902.

59. C. Truesdell and W. Noll, The non-linear field theories of mechanics: in Handbuch der Physik, Vol. III/3, edited by S. Flugge, (Springer-Verlag, Berlin, 1965), pp.1- 
662.

60. D. C. Wallace, Thermoelastic theory of stressed crystals and higher order elastic constants: in Solid State Physics, vol.25, edited by H. Ehrenreich, F. Seitz, and D. Turnbull, (Academic, New York, 1970), pp. 301-404.

61. J. H. Cantrell, Elastic constants of solids and fluids with initial pressure via a unified approach based on equations-of-state, Ultrasonics 54,1323-1331 (2014).

62. P. Ehrenfest, Adiabatic invariants and the theory of quanta, Philos. Mag. 33, 500-513 (1917).

63. M. Greenspan, Simple derivation of the Boltzmann-Ehrenfest Adiabatic Principle, J. Acoust. Soc. Am. 27, 34-35 (1955).

64. H. Goldstein, Classical Mechanics, $2^{\text {nd }}$ edition, (Addison-Wesley, Reading, Massachusetts, 1980), pp 1-672.

65. J. H. Cantrell, Acoustic rectification and the virial theorem, J. Phys. A: Math. Gen. 26, L673 (1993).

66. H. F. Pollard, Sound Waves in Solids, (Pion, London, 1977), pp. 1-366. 


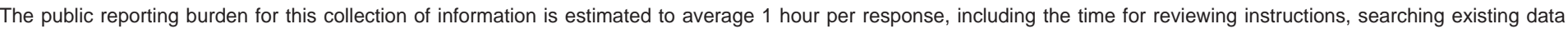

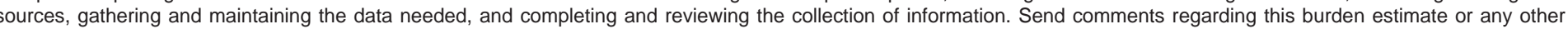

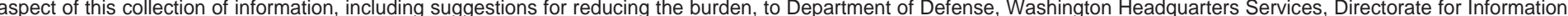

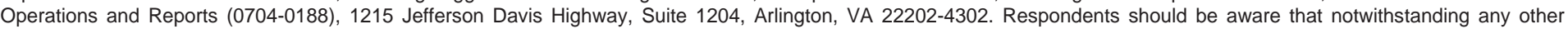
provision of law, no person shall be subject to any penalty for failing to comply with a collection of information if it does not display a currently valid OMB control number. PLEASE DO NOT RETURN YOUR FORM TO THE ABOVE ADDRESS.

\begin{tabular}{|l|c|c|c|}
\hline $\begin{array}{l}\text { 1. REPORT DATE (DD-MM-YYYY) } \\
1-04-2018\end{array}$ & $\begin{array}{c}\text { 2. REPORT TYPE } \\
\text { Technical Memorandum }\end{array}$ & 3. DATES COVERED (FrOm - To) \\
\hline 4. TITLE AND SUBTITLE & 5a. CONTRACT NUMBER
\end{tabular}

Acoustic Radiation Pressure

5b. GRANT NUMBER

5c. PROGRAM ELEMENT NUMBER

6. AUTHOR(S)

5d. PROJECT NUMBER

Cantrell, John H.

5e. TASK NUMBER

5f. WORK UNIT NUMBER

7. PERFORMING ORGANIZATION NAME(S) AND ADDRESS(ES)

826611.04.07.02

8. PERFORMING ORGANIZATION REPORT NUMBER

NASA Langley Research Center

Hampton, VA 23681-2199

L-20907

9. SPONSORING/MONITORING AGENCY NAME(S) AND ADDRESS(ES)

10. SPONSOR/MONITOR'S ACRONYM(S)

National Aeronautics and Space Administration

NASA

Washington, DC 20546-0001

11. SPONSOR/MONITOR'S REPORT NUMBER(S)

NASA-TM-2018-219806

\section{DISTRIBUTIONIAVAILABILITY STATEMENT}

Unclassified

Subject Category 71

Availability: NASA STI Program (757) 864-9658

\section{SUPPLEMENTARY NOTES}

\section{ABSTRACT}

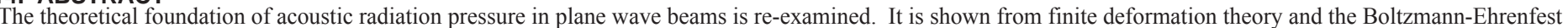

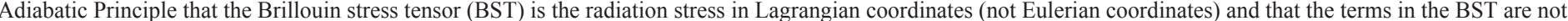

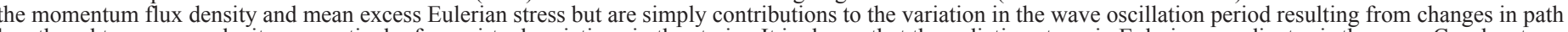

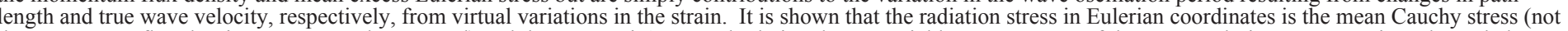

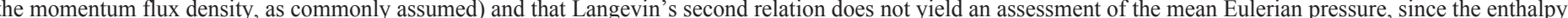

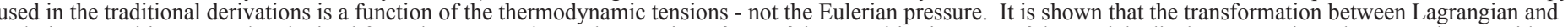

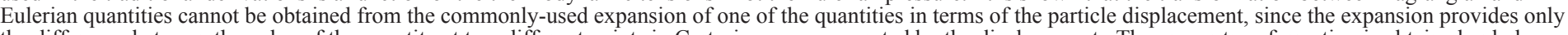

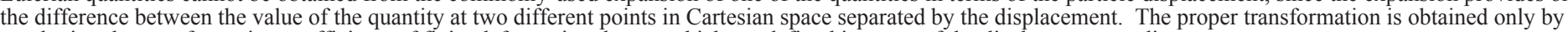
employing the transformation coefficients of finite deformation theory, which are defined in terms of the displacement gradients.

\section{SUBJECT TERMS}

Acoustic radiation pressure; Adiabatic invariance; Finite deformation theory; Laterally confined and un-confined waves

16. SECURITY CLASSIFICATION OF:

a. REPORT

b. ABSTRACT

c.

$\mathrm{U}$

$\mathrm{U}$
17. LIMITATION OF ABSTRACT

UU
18. NUMBER OF
PAGES

59 19a. NAME OF RESPONSIBLE PERSON

STI Help Desk (email: help@sti.nasa.gov)

19b. TELEPHONE NUMBER (Include area code) (757) 864-9658
U 